\title{
Union Averaged Operators with Applications to Proximal Algorithms for Min-Convex Functions
}

\author{
Minh N. Dao* \\ Matthew K. Tam ${ }^{\dagger}$
}

October 17, 2018

\begin{abstract}
In this paper, we introduce and study a class of structured set-valued operators which we call union averaged nonexpansive. At each point in their domain, the value of such an operator can be expressed as a finite union of single-valued averaged nonexpansive operators. We investigate various structural properties of the class and show, in particular, that is closed under taking unions, convex combinations, and compositions, and that their fixed point iterations are locally convergent around strong fixed points. We then systematically apply our results to analyze proximal algorithms in situations where union averaged nonexpansive operators naturally arise. In particular, we consider the problem of minimizing the sum two functions where the first is convex and the second can be expressed as the minimum of finitely many convex functions.
\end{abstract}

\section{Mathematics Subject Classification (MSC 2010): $90 \mathrm{C} 26 \cdot 47 \mathrm{H} 10 \cdot 47 \mathrm{H} 04$}

Keywords: admissible control $\cdot$ averaged operator $\cdot$ fixed point iteration $\cdot$ local convergence $\cdot$ proximal algorithms $\cdot$ set-valued map

\section{Introduction}

The notion of an averaged nonexpansive operator is one which nicely balances two properties of importance in the context of fixed point algorithms, namely, usefulness and applicability. In this context, usefulness is meant in the sense that algorithms based on such operators are provably convergent, for instance, by appealing to Opial-type results [1,2], and applicability is meant in sense that the class of averaged nonexpansive is significantly rich so as to include many commonly encountered operators. Indeed, the class includes all firmly nonexpansive operators as well as their convex combinations and compositions [3, Section 4.5]. For further information on averaged operators, the reader is referred to $[4,3,1]$ and the references therein.

In many applications, particularly those involving some of kind of nonconvexity, the involved algorithmic operator is not averaged nonexpansive. Nevertheless, it is sometimes still the case that some underlying averaged nonexpansive structure which can be exploited is present. A notable example is provided by sparsity constrained optimization in which the feasible region is a lower-level set of the $\ell_{0^{-}}$psuedo norm. This set can be naturally expressed as the union of a finite number of "sparsity subspaces". Consequently, at each point in the space, its metric projector can be expressed as the union a subset of the averaged nonexpansive projectors onto these subspaces, although the projector onto the lower-set itself is not averaged nonexpansive. Indeed, this type of decomposability was consider in [5] which was, in turn, inspired by [6].

In this work, we aim to exploit structure of the aforementioned type. More precisely, we consider a class of set-valued operators which we call union averaged nonexpansive (as well as the notion of a union nonexpansive operator). At each point in the ambient space, the value of these operators can be described as a union of single-valued averaged nonexpansive operators from a finite family. A related notion, union paracontracting operators, was previously studied by the second author in [5]. A significant short-coming of the class of union paracontracting operators is that they are, in general, not closed under

\footnotetext{
*CARMA, University of Newcastle, Callaghan, NSW 2308, Australia. E-mail: daonminh@gmail.com

${ }^{\dagger}$ Institut für Numerische und Angewandte Mathematik, Universität Göttingen, 37083 Göttingen, Germany. E-mail: m.tam@math.uni-goettingen.de
} 
operators taking convex combination and compositions, thus making it more difficult to determine if a given operators belong to the class. The situation is remedied by union average nonexpansiveness.

The remainder of this paper is organized as follows. We begin, in Section 2, by recalling the necessary mathematical preliminaries. In Section 3 we introduce the notion of union averaged nonexpansive operators and study their closure and fixed point properties (Proposition $3.2 \& 3.3$ ). In Section 4, we provide a variation of [7] (Theorem 4.1) and, as a corollary, deduce local convergence for union (averaged) nonexpansive maps around their strong fixed points (Theorem 4.2). In Section 5, we introduce and study functions which can be expressed as the minimum of finitely many convex functions; we term these functions min-convex before concluding, in Section 6 with a systematic study of proximal algorithms applied to minimization of min-convex problems including various projection algorithms, the proximal point algorithm, forward-backward splitting and Douglas-Rachford splitting.

\section{Preliminaries}

In this section, we recall the necessary preliminaries for the subsequent sections. Unless stated otherwise, throughout this work we assume that

$$
\mathrm{X} \text { is a (real) finite-dimensional Hilbert space }
$$

with inner-product $\langle\cdot, \cdot\rangle$ and induced norm $\|\cdot\|$.

In order to introduce the two new classes of structured set-valued operators in Definition 3.1, we first recall the definitions and basic properties of their single-valued counterparts. The term "averaged" originally appeared in [4].

Definition 2.1 (Averaged nonexpansive operators). A single-valued operator $T: X \rightarrow X$ is said to be nonexpansive if

$$
\forall x, y \in X, \quad\|T x-T y\| \leq\|x-y\|,
$$

and $\alpha$-averaged nonexpansive if $\alpha \in(0,1)$ and

$$
\forall x, y \in X, \quad\|T x-T y\|^{2}+\frac{1-\alpha}{\alpha}\|(\operatorname{Id}-T) x-(\operatorname{Id}-T) y\|^{2} \leq\|x-y\|^{2} .
$$

We say $T$ is averaged nonexpansive if there is an $\alpha \in(0,1)$ such that $T$ is $\alpha$-averaged nonexpansive.

It follows immediately from the above definition that every averaged nonexpansive operator is nonexpansive. The precise relationship between the two classes is given in the following proposition.

Proposition 2.1 (Characterizations of averaged nonexpansiveness). Let $T: X \rightarrow X$ be an operator and let $\alpha \in(0,1)$. The following assertions are equivalent.

(a) $T$ is $\alpha$-averaged nonexpansive.

(b) $T=(1-\alpha) \mathrm{Id}+\alpha R$ for some nonexpansive operator $R: X \rightarrow X$.

(c) $(1-1 / \alpha) \operatorname{Id}+(1 / \alpha) T$ is nonexpansive.

Proof. Follows by combining [3, Definition 4.33 and Proposition 4.35].

The following proposition shows that the classes of nonexpansive and averaged nonexpansive operators are both closed under taking convex combination and under compositions. Such properties are of interest because they provide a way to verify that a given operator is averaged nonexpansive in the case that it can be represented in terms of simpler operators whose averaged nonexpansiveness can be more easily checked.

Proposition 2.2 (Convex combinations and compositions). Let $J:=\{1, \ldots, m\}$ and let $T_{j}: X \rightarrow X$ be $\alpha_{j}$-averaged nonexpansive (resp. nonexpansive) for each $j \in J$. Then the following assertions hold.

(a) $\sum_{j \in J} \omega_{j} T_{j}$ is $\alpha$-averaged nonexpansive with

$$
\alpha:=\sum_{j \in J} \omega_{j} \alpha_{j}
$$

(resp. nonexpansive) whenever $\left(\omega_{j}\right)_{j \in J} \subseteq \mathbb{R}_{++}$with $\sum_{j \in J} \omega_{j}=1$. 
(b) $T_{m} \circ \cdots \circ T_{2} \circ T_{1}$ is $\alpha$-averaged nonexpansive with

$$
\alpha:=\left(1+\left(\sum_{j \in J} \frac{\alpha_{j}}{1-\alpha_{j}}\right)^{-1}\right)^{-1}
$$

(resp. nonexpansive).

Proof. See [8, Propositions $2.2 \& 2.5]$. Note also that Definition 2.1 for averaged operators appeared in [1, Definition 2.2.14] under the name $\nu$-firmly nonexapnsive operators with $\nu$ taken to be $(1-\alpha) / \alpha$. Appealing to the connection given in [1, Corollary 2.2.17], the conclusion can be also deduced from [1, Theorems 2.2.35 \& 2.2.42].

The second notion that will be used in Definition 3.1 is that of an outer semicontinuous map [9, Section $3 \mathrm{~B}]$. In what follows, we recall its definition and basic properties.

Definition 2.2 (Outer semicontinuity). Let $X$ and $Y$ be Hilbert spaces. A set-valued map $\phi: X \rightrightarrows Y$ is outer semicontinuous (osc) at $\bar{x}$ if

$$
\phi(\bar{x}) \supseteq \operatorname{Limsup}_{x \rightarrow \bar{x}} \phi(x):=\left\{y \in Y: \exists x_{n} \rightarrow \bar{x}, \exists y_{n} \rightarrow y \text { with } y_{n} \in \phi\left(x_{n}\right)\right\} .
$$

That is, the limit supremum is understood in the sense of the Painlevé-Kuratowski outer limit on $X \times Y$.

Proposition 2.3 (Cartesian products). Let $J:=\{1, \ldots, m\}$ and let $\phi_{j}: X \rightrightarrows Y_{j}$ be osc for each $j \in J$. Then the mapping $\phi: X \rightrightarrows Y_{1} \times \cdots \times Y_{m}$ defined by

$$
x \mapsto \phi(x):=\phi_{1}(x) \times \cdots \times \phi_{m}(x)
$$

is also osc.

Proof. Let $\bar{x} \in X$ and consider sequences $x_{n} \rightarrow \bar{x}$ and $y_{n}:=\left(y_{1 n}, \ldots, y_{m n}\right) \rightarrow y:=\left(y_{1}, \ldots, y_{m}\right)$ and $y_{n} \in \phi\left(x_{n}\right)$, or equivalently, $y_{j n} \in \phi_{j}\left(x_{n}\right)$ for every $j \in J$. Since each $\phi_{j}$ is osc, it holds that $y_{j} \in \phi_{j}(\bar{x})$, and hence $y \in \phi(\bar{x})=\phi_{1}(\bar{x}) \times \cdots \times \phi_{m}(\bar{x})$.

Proposition 2.4. Let $\phi: X \rightrightarrows Y$ be osc, $I$ be a nonempty finite index set, $\left\{T_{i}\right\}_{i \in I}$ be a collection of continuous single-valued operators on $X$, and $\varphi: X \rightrightarrows I$ be osc. Then the mapping $\psi: X \rightrightarrows Y$ is osc where $\psi$ is defined by

$$
x \mapsto \psi(x):=\left\{\phi\left(T_{i}(x)\right): i \in \varphi(x)\right\} .
$$

Consequently, if $T: X \rightarrow X$ is continuous, then $\phi \circ T$ is osc.

Proof. Let $\bar{x} \in X$ and consider sequences $x_{n} \rightarrow \bar{x}$ and $y_{n} \rightarrow y$ with $y_{n} \in \psi\left(x_{n}\right)$. By definition, $y_{n} \in$ $\phi\left(T_{i_{n}}\left(x_{n}\right)\right)$ for some $i_{n} \in \varphi\left(x_{n}\right) \subseteq I$. Since $I$ is finite, by passing to a subsequence, we may assume that $i_{n}=i \in I$ for all $n$. Then the osc of $\varphi$ implies that $i \in \varphi(\bar{x})$, the continuity of $T_{i}$ implies that $T_{i}\left(x_{n}\right) \rightarrow T_{i}(\bar{x})$. As $\phi$ is osc and $y_{n} \in \phi\left(T_{i}\left(x_{n}\right)\right)$, it follows that $y \in \phi\left(T_{i}(\bar{x})\right) \subseteq \psi(\bar{x})$.

\section{Unions of averaged operators}

In this section, we introduce the classes of operators which are the main object of study in this work and investigate their properties. We begin with their definition.

Definition 3.1 (Union averaged nonexpansive operators). A set-valued operator $T: X \rightrightarrows X$ is said to be union $\alpha$-averaged nonexpansive (resp. union nonexpansive) if $T$ can be expressed in the form

$$
\forall x \in X, \quad T(x)=\left\{T_{i}(x): i \in \varphi(x)\right\},
$$

where $I$ is a finite index set, $\left\{T_{i}\right\}_{i \in I}$ is a collection of $\alpha$-averaged nonexpansive (resp. nonexpansive) operators on $X$, and $\varphi: X \rightrightarrows I$, called an active selector, is an osc operator with nonempty values.

As before, we say $T$ is union averaged nonexpansive if there is an $\alpha \in(0,1)$ such that $T$ is union $\alpha$-averaged nonexpansive.

In order to demonstrate as situation in which union averaged nonexpansiveness naturally arises, we state the following example which we shall return to in Section 5. 
Example 3.1 (Sparsity projectors). Let $X=\mathbb{R}^{n}$ and $s \in\{0,1, \ldots, n-1\}$. A common approach in sparsity optimization involves minimization over the nonconvex sparsity constraint set

$$
C:=\left\{x \in \mathbb{R}^{n}:\|x\|_{0} \leq s\right\}
$$

where $\|\cdot\|_{0}$ denotes the $\ell_{0}$-functional which counts the number of nonzero entries in a vector. By denoting $\mathcal{I}:=\left\{I \in 2^{\{1,2, \ldots, n\}}:|I|=s\right\}$, the set $C$ can be naturally expressed as a union of nonempty subspaces as

$$
C=\bigcup_{I \in \mathcal{I}} C_{I} \text { where } C_{I}:=\left\{x \in \mathbb{R}^{n}: x_{i} \neq 0 \text { only if } i \in I\right\}
$$

In Proposition 6.1(a), we shall show that nearest point projector onto $C$ is union $1 / 2$-averaged nononexpansive with

$$
P_{C}(x):=\{c \in C:\|x-c\|=\mathrm{d}(c, C)\}=\left\{P_{C_{I}}(x): I \in \varphi(x)\right\}
$$

where $\varphi(x):=\left\{I \in \mathcal{I}: \min _{i \in I}\left|x_{i}\right| \geq \max _{i \notin I}\left|x_{i}\right|\right\}$.

The following proposition is the union averaged nonexpansive analogue of Proposition 2.1 and offers equivalent characterizations of union averaged nonexpansiveness. In what follows, the sum of two or more sets is understood in the sense of the Minkowski sum.

Proposition 3.1 (Equivalent characterizations of union averaged nonexpansiveness). Let $T: X \rightrightarrows X$ be a set-valued operator and let $\alpha \in(0,1)$. The following assertions are equivalent.

(a) $T$ is union $\alpha$-averaged nonexpansive.

(b) $T=(1-\alpha) \mathrm{Id}+\alpha R$ for some union nonexpansive operator $R$ : $X \rightrightarrows X$.

(c) $(1-1 / \alpha) \operatorname{Id}+(1 / \alpha) T$ is union nonexpansive.

Proof. Follows by combining Definition 3.1 with Proposition 2.1.

A class of operators related to those in Definition 3.1, the class of union paracontracting operators, was introduced in [5]. Recall that a single-valued operator $S: X \rightarrow X$ is paracontracting if it is continuous and strictly quasi-nonexpansive, that is,

$$
\forall x \in X \backslash \operatorname{Fix} S, \forall y \in \operatorname{Fix} S, \quad\|S(x)-y\|<\|x-y\| .
$$

An operator $T: X \rightrightarrows X$ of the form (1) is union paracontracting if $\left\{T_{i}\right\}_{i \in I}$ is instead a collection of paracontracting operators.

In general, convex combinations and compositions of paracontracting operators need not stay paracontracting except when the individual operators share a common fixed points; see [1, Theorem 2.1.26 and Corollary 2.1.29]. Consequently, the same is true of union paracontracting operators. As the following proposition shows, this shortcomming is rectified by using averaged nonexpansive operators in place of paracontracting.

Proposition 3.2 (Unions, combinations and compositions). Let $J:=\{1, \ldots, m\}$ and let $T_{j}: X \rightrightarrows X$ be union $\alpha_{j}$-averaged nonexpansive (resp. union nonexpansive) for each $j \in J$. Then the following assertions hold.

(a) $T: X \rightrightarrows X$ defined by $x \mapsto T(x):=\cup_{j \in J} T_{j}(x)$ is union $\alpha$-averaged nonexpansive with

$$
\alpha:=\max _{j \in J} \alpha_{j}
$$

(resp. union nonexpansive).

(b) $\sum_{j \in J} \omega_{j} T_{j}$ is union $\alpha$-averaged nonexpansive with

$$
\alpha:=\sum_{j \in J} \omega_{j} \alpha_{j}
$$

(resp. union nonexpansive) whenever $\left(\omega_{j}\right)_{j \in J} \subseteq \mathbb{R}_{++}$with $\sum_{j \in J} \omega_{j}=1$. 
(c) $T_{m} \circ \cdots \circ T_{2} \circ T_{1}$ is union $\alpha$-averaged nonexpansive with

$$
\alpha:=\left(1+\left(\sum_{j \in J} \frac{\alpha_{j}}{1-\alpha_{j}}\right)^{-1}\right)^{-1}
$$

(resp. union nonexpansive).

Proof. First note that as $T_{j}$ is union $\alpha_{j}$-averaged nonexpansive (resp. union nonexpansive), by definition, there exists a finite index set $I_{j}$, an osc map $\varphi_{j}: X \rightrightarrows I_{j}$ and a collection of $\alpha_{j}$-averaged nonexpansive (resp. nonexpansive) operators $\left\{T_{j, i_{j}}\right\}_{i_{j} \in I_{j}}$ on $X$ such that $T_{j}$ can be expressed as

$$
\forall x \in X, \quad T_{j}(x)=\left\{T_{j, i_{j}}(x): i_{j} \in \varphi_{j}(x)\right\} .
$$

(a): For all $x \in X$, the definition of $T$ yields

$$
T(x)=\bigcup_{j \in J} T_{j}(x)=\left\{T_{j, i_{j}}(x): j \in J, i_{j} \in \varphi_{j}(x)\right\}=\left\{T_{j, i}(x):(j, i) \in \varphi(x)\right\}
$$

where $\varphi: X \rightrightarrows J \times \cup_{j \in J} I_{j}$ is defined as

$$
\varphi(x):=\left\{(j, i): j \in J, i \in \varphi_{j}(x)\right\} .
$$

Note that $T_{j, i}$ is $\alpha$-averaged nonexpansive (resp. nonexpansive) for each $j \in J$ and each $i \in I_{j}$ since $\alpha \geq \alpha_{j}$, hence we only need to prove osc of $\varphi$. To this end, consider $x_{n} \rightarrow x$ in $X$ and $\left(j_{n}, i_{n}\right) \rightarrow(j, i)$ in $J \times \cup_{j \in J} I_{j}$ with $\left(j_{n}, i_{n}\right) \in \varphi\left(x_{n}\right)$. Since $J$ is finite and $j_{n} \rightarrow j$, there exists $n_{0} \in \mathbb{N}$ such that $j_{n}=j$ for all $n \geq n_{0}$, and we therefore have $i_{n} \in \varphi_{j_{n}}(x)=\varphi_{j}(x)$ for $n \geq n_{0}$. Outer semicontinuity of $\varphi_{j}$ implies that $i \in \varphi_{j}(x)$ and hence that $(j, i) \in \varphi(x)$. Consequently, $\varphi$ is osc, and $T$ is thus union $\alpha$-averaged nonexpansive (resp. union nonexpansive).

(b): The definition of the Minkowski sum gives that

$$
\sum_{j \in J} \omega_{j} T_{j}(x)=\sum_{j \in J} \omega_{j}\left\{T_{j, i_{j}}(x): i_{j} \in \varphi_{j}(x)\right\}=\left\{\sum_{j \in J} \omega_{j} T_{j, i_{j}}(x):\left(i_{1}, \ldots, i_{m}\right) \in \varphi(x)\right\}
$$

where $\varphi: X \rightrightarrows I_{1} \times \cdots \times I_{m}$ is defined as $\varphi(x):=\varphi_{1}(x) \times \cdots \times \varphi_{m}(x)$. Outer semicontinuity of $\varphi$ follows from Proposition 2.3. Since $T_{j, i_{j}}$ is $\alpha_{j}$-averaged nonexpansive (resp. nonexpansive) for each $i_{j} \in I_{j}$ and $j \in J$, Proposition 2.2(a) implies that $\sum_{j \in J} \omega_{j} T_{j, i_{j}}$ is $\alpha$-averaged nonexpansive, for all $\left(i_{1}, \ldots, i_{m}\right) \in I_{1} \times \cdots \times I_{m}$, with $\alpha$ given by (2) (resp. union nonexpansive) which completes the proof.

(c): Using the definition of operator composition, we deduce that

$$
\left(T_{m} \circ \cdots \circ T_{1}\right)(x)=\left\{\left(T_{m, i_{m}} \circ \cdots \circ T_{1, i_{1}}\right)(x):\left(i_{1}, \ldots, i_{m}\right) \in \varphi(x)\right\}
$$

where $\varphi: X \rightrightarrows I_{1} \times \cdots \times I_{m}$ is given by

$$
\varphi(x):=\left\{\left(i_{1}, \ldots, i_{m}\right): i_{1} \in \varphi_{1}(x), i_{2} \in\left(\varphi_{2} \circ T_{1, i_{1}}\right)(x), \ldots, i_{m} \in\left(\varphi_{m} \circ T_{m-1, i_{m-1}} \circ \cdots \circ T_{1, i_{1}}\right)(x)\right\} .
$$

To show that $\varphi$ is osc, consider sequences $x_{n} \rightarrow x$ and $i_{n}=\left(i_{1, n}, \ldots, i_{m, n}\right) \rightarrow i=\left(i_{1}, \ldots, i_{m}\right)$ such that $i_{n} \in \varphi\left(x_{n}\right)$. Since $I_{1} \times \cdots \times I_{m}$ is finite, there exists an $n_{0} \in \mathbb{N}$ such that $i_{n}=i$ for all $n \geq n_{0}$. Then, for all $n \geq n_{0}, i=\left(i_{1}, \ldots, i_{m}\right) \in \varphi\left(x_{n}\right)$, that is,

$$
i_{j+1} \in \begin{cases}\varphi_{1}\left(x_{n}\right) & \text { if } j=0 \\ \left(\varphi_{j+1} \circ T_{j, i_{j}} \circ \cdots \circ T_{1, i_{1}}\right)\left(x_{n}\right) & \text { if } j \in J \backslash\{m\} .\end{cases}
$$

Then, osc of $\varphi$ follows by combining Propositions $2.3 \& 2.4$, noting that $T_{j, i_{j}}$ is continuous, for each $j \in J \backslash\{m\}$. Finally, since $T_{j, i_{j}}$ is $\alpha_{j}$-averaged nonexpansive (resp. nonexpansive) for each $i_{j} \in I_{j}$ and $j \in J$, Proposition 2.2(b) implies that $\left(T_{m, i_{m}} \circ \cdots \circ T_{1, i_{1}}\right)$ is $\alpha$-averaged nonexpansive with $\alpha$ given by (3) (resp. nonexpansive) which completes the proof.

For set-valued operators such as those introduced in Definition 3.1, we distinguish two different notions for fixed points which are both the same in the single-valued case. The fixed point set of $T$ is denoted by Fix $T:=\{x: x \in T(x)\}$, and the strong fixed point set of $T$ is given by Fix $T:=\{x: T(x)=\{x\}\}$. 
In the following proposition, we take a closer look the structure of a class of set-valued operators which includes union averaged nonexpansive operators as a special case. Given a set-valued operator $T: X \rightrightarrows X$, its single-valued set, denoted by

$$
\text { Sing } T:=\{x \in X: T(x) \text { is a singleton }\},
$$

is the set of points at which $T$ is single-valued.

Proposition 3.3 (Active selectors, fixed points, single-valuedness). Let $T: X \rightrightarrows X$ be a set-valued operator given by

$$
\forall x \in X, \quad T(x)=\left\{T_{i}(x): i \in \varphi(x)\right\}
$$

where $I$ is a finite index set, $\varphi: X \rightrightarrows I$ is an osc operator with nonempty values, and $\left\{T_{i}\right\}_{i \in I}$ is a collection of single-valued operators on $X$. The following assertions hold.

(a) For each $i \in I$, the set $\varphi^{-1}(i)$ is closed. The space $X$ can be represented as

$$
X=\bigcup_{i \in I} \varphi^{-1}(i)
$$

(b) $x \in \operatorname{Fix} T$ if and only if there exists $i \in I$ such that $x \in \varphi^{-1}(i) \cap \operatorname{Fix} T_{i}$. Consequently, we have

$$
\operatorname{Fix} T=\bigcup_{i \in I}\left[\varphi^{-1}(i) \cap \operatorname{Fix} T_{i}\right]
$$

(c) $x \in \mathbf{F i x} T$ if and only if

$$
x \in \bigcap_{i \in \varphi(x)} \operatorname{Fix} T_{i}=\bigcap_{i \in \varphi(x)}\left[\varphi^{-1}(i) \cap \operatorname{Fix} T_{i}\right] .
$$

(d) The strong fixed point set is the intersection of the fixed point set and the single-valued set, that is,

$\operatorname{Fix} T=\operatorname{Fix} T \cap \operatorname{Sing} T$.

(e) If in addition $\left\{T_{i}\right\}_{i \in I}$ is a collection of continuous operators, then $T$ is ocs. Consequently,

$$
\operatorname{Sing} T=\left\{x \in X: \operatorname{Limsup}_{x^{\prime} \rightarrow x} T\left(x^{\prime}\right) \text { is singleton }\right\} \text {. }
$$

Proof. (a): Let $i \in I$ and consider a sequence $\left(x_{n}\right)_{n \in \mathbb{N}} \subseteq \varphi^{-1}(i)$ such that $x_{n} \rightarrow \bar{x}$. The definition of the inverse implies that $i \in \varphi\left(x_{n}\right)$ for all $n \in \mathbb{N}$ and, since $\varphi$ is osc, it holds that $i \in \varphi(\bar{x})$, which shows that $\varphi^{-1}(i)$ is closed and proves the first claim. The fact that (4) follows from the assumption that $\varphi(x) \neq \varnothing$ for all $x \in X$.

(b): $x \in \operatorname{Fix} T \Longleftrightarrow \exists i \in \varphi(x)$ such that $x \in \operatorname{Fix} T_{i} \Longleftrightarrow \exists i \in I$ such that $x \in \varphi^{-1}(i) \cap$ Fix $T_{i}$.

(c): $x \in \operatorname{Fix} T \Longleftrightarrow x \in \operatorname{Fix} T_{i}$ for all $i \in \varphi(x) \Longleftrightarrow x \in \varphi^{-1}(i) \cap \operatorname{Fix} T_{i}$ for all $i \in \varphi(x)$.

(d): Immediate from the respective definitions.

(e): Fix $x \in X$. We first show that $T$ is osc. To this end, take $x_{n} \rightarrow x$ and $y_{n} \rightarrow y$ with $y_{n} \in T\left(x_{n}\right)$. Then the definition of $T$ ensures the existence of sequence $i_{n} \in \varphi\left(x_{n}\right)$ such that $y_{n}=T_{i_{n}}\left(x_{n}\right) \in T\left(x_{n}\right)$. Using the pigeonhole princple, we pass to a subsequence so that $y_{k_{n}}=T_{i}\left(x_{k_{n}}\right)$ for fixed $i \in I$. The osc of $\varphi$ implies that $i \in \varphi(x)$ and continuity of $T_{i}$ gives

$$
y=\lim _{n \rightarrow \infty} y_{k_{n}}=\lim _{n \rightarrow \infty} T_{i}\left(x_{k_{n}}\right)=T_{i}(x) \in T(x),
$$

which proves osc. The claimed formula for the singleton set follows since $x \in \operatorname{Sing} T \Longleftrightarrow T(x)$ is singleton, and osc implies that $T(x)=\operatorname{Limsup}_{x^{\prime} \rightarrow x} T\left(x^{\prime}\right)$. 


\section{Convergence of fixed point algorithms}

In this section, we prove our main result regarding local convergence of fixed point iterations based on union averaged nonexpansive operators. More precisely, given a union $\alpha$-averaged nonexpansive $T: X \rightrightarrows$ $X$ defined by $x \mapsto T(x):=\left\{T_{i}(x): i \in \varphi(x)\right\}$, we study the behaviour of iterations of the form

$$
x_{0} \in X \quad \text { and } \quad \forall n \in \mathbb{N}, x_{n+1} \in\left(\left(1-\lambda_{n}\right) \operatorname{Id}+\lambda_{n} T\right)\left(x_{n}\right),
$$

where $\left(\lambda_{n}\right)_{n \in \mathbb{N}} \subseteq(0,1 / \alpha]$ and Id denotes the identity operator. To do so, we first study the following closely related iterations given by

$$
x_{0} \in X \quad \text { and } \quad \forall n \in \mathbb{N}, x_{n+1} \in\left(\left(1-\lambda_{n}\right) \operatorname{Id}+\lambda_{n} T_{i_{n}}\right)\left(x_{n}\right) \text {, }
$$

where each element in $I$ appears infinitely often in the sequence $\left(i_{n}\right)_{n \in \mathbb{N}} \subseteq I$.

The condition that each element in $I$ must appear infinitely often in a sequence is $\left(i_{n}\right)_{n \in \mathbb{N}} \subseteq I$ is generalized in the following definition.

Definition 4.1 (Admissible sequences). Let $I$ and $I^{*}$ be nonempty finite sets with $I^{*} \subseteq I$. A sequence $\left(i_{n}\right)_{n \in \mathbb{N}} \subseteq I$ is admissible in $I^{*}$ if every element of $I^{*}$ appears infinitely often in $\left(i_{n}\right)_{n \in \mathbb{N}}$.

\subsection{Krasnosel'skiı̈-Mann iterations with admissible control}

We begin with the following variation of [7, Theorem 1]. Although the proof is straightforward, we include it for the sake of completeness.

Theorem 4.1 (Krasnosel'skiǔ-Mann iterations with admissible control). Let I be a finite index set and let $\left\{T_{i}\right\}_{i \in I}$ be a collection of nonexpansive operators on $X$ with a common fixed point. Define a sequence $\left(x_{n}\right)_{n \in \mathbb{N}}$ with starting point $x_{0} \in X$ according to

$$
\forall n \in \mathbb{N}, \quad x_{n+1}:=\left(1-\lambda_{n}\right) x_{n}+\lambda_{n} T_{i_{n}}\left(x_{n}\right),
$$

where $\left(i_{n}\right)_{n \in \mathbb{N}}$ is admissible in $I$, and $\left(\lambda_{n}\right)_{n \in \mathbb{N}}$ is in $(0,1]$ with $\liminf _{n \rightarrow \infty} \lambda_{n}\left(1-\lambda_{n}\right)>0$. Then $\left(x_{n}\right)_{n \in \mathbb{N}}$ converges to a point $\bar{x} \in \cap_{i \in I}$ Fix $T_{i}$.

Proof. Since $\liminf \inf _{n \rightarrow \infty} \lambda_{n}\left(1-\lambda_{n}\right)>0$, there exists $n_{0} \in \mathbb{N}$ such that $\inf _{n>n_{0}} \lambda_{n}\left(1-\lambda_{n}\right)>0$. By relabeling if necessary, we may assume without loss of generality that $\varepsilon:=\inf _{n \in \mathbb{N}} \lambda_{n}\left(1-\lambda_{n}\right)>0$. Let $x \in \cap_{i \in I}$ Fix $T_{i}$ be arbitrary. Then, for all $n \in \mathbb{N}$, nonexpansiveness of $T_{i_{n}}$ yields

$$
\begin{aligned}
\left\|x_{n+1}-x\right\|^{2} & =\left\|\left(1-\lambda_{n}\right)\left(x_{n}-x\right)+\lambda_{n}\left(T_{i_{n}}\left(x_{n}\right)-x\right)\right\|^{2} \\
& =\left(1-\lambda_{n}\right)\left\|x_{n}-x\right\|^{2}+\lambda_{n}\left\|T_{i_{n}}\left(x_{n}\right)-x\right\|^{2}-\lambda_{n}\left(1-\lambda_{n}\right)\left\|x_{n}-T_{i_{n}}\left(x_{n}\right)\right\|^{2} \\
& \leq\left\|x_{n}-x\right\|^{2}-\varepsilon\left\|x_{n}-T_{i_{n}}\left(x_{n}\right)\right\|^{2} .
\end{aligned}
$$

It follows that $\left(\left\|x_{n}-x\right\|^{2}\right)_{n \in \mathbb{N}}$ is monotone nonincreasing, and hence convergent. Consequently, the sequence $\left(x_{n}\right)_{n \in \mathbb{N}}$ is bounded and

$$
x_{n}-T_{i_{n}}\left(x_{n}\right) \rightarrow 0 \text { as } n \rightarrow \infty .
$$

Now, let $\bar{x} \in X$ be a cluster point of $\left(x_{n}\right)_{n \in \mathbb{N}}$. Then $\left(x_{n}\right)_{n \in \mathbb{N}}$ contains a convergent subsequence, say $\left(x_{k_{n}}\right)_{n \in \mathbb{N}}$, with limit $\bar{x}$. We claim that $\bar{x}$ is an element of $\cap_{i \in I}$ Fix $T_{i}$. To this end suppose, by way of a contradiction, that $\bar{x} \notin \cap_{i \in I}$ Fix $T_{i}$. By passing to a further subsequence if necessary, we deduce that the sequence $\left(t_{n}\right)_{n \in \mathbb{N}}$ defined by

$$
t_{n}:=\min \left\{p \in\left\{k_{n}, \ldots, k_{n+1}-1\right\}: \bar{x} \notin \operatorname{Fix} T_{i_{p}}\right\},
$$

is well defined. Since $I$ is finite, by passing to yet another subsequence if necessary, we may assume that $i_{t_{n}}=\ell$ for all $n \in \mathbb{N}$ for some fixed index $\ell \in I$. Together with (6), we deduce that

$$
x_{t_{n}} \rightarrow \bar{x} \text { and }\left(\mathrm{Id}-T_{\ell}\right)\left(x_{t_{n}}\right) \rightarrow 0 \quad \text { as } n \rightarrow \infty,
$$

which, due to nonexpansiveness of $T_{\ell}$, implies that $\bar{x} \in \mathrm{Fix} T_{\ell}$. Thus a contradiction is obtained, and conclude that $\bar{x} \in \cap_{i \in I} \operatorname{Fix} T_{i}$. We then have that $\left(\left\|x_{n}-\bar{x}\right\|\right)_{n \in \mathbb{N}}$ is monotone nonincreasing and $\| x_{k_{n}}-$ $\bar{x} \| \rightarrow 0$, and thus the conclusion follows. 
Corollary 4.1. Let $I$ be a finite index set and let $\left\{T_{i}\right\}_{i \in I}$ be $\alpha_{i}$-averaged nonexpansive operators on $X$ with a common fixed point. Define a sequence $\left(x_{n}\right)_{n \in \mathbb{N}}$ with starting point $x_{0} \in X$ according to

$$
\forall n \in \mathbb{N}, \quad x_{n+1}=\left(1-\lambda_{n}\right) x_{n}+\lambda_{n} T_{i_{n}}\left(x_{n}\right),
$$

where $\left(i_{n}\right)_{n \in \mathbb{N}}$ is an admissible sequence in $I$, and $\left(\lambda_{n}\right)_{n \in \mathbb{N}}$ is a sequence satisfying $\lambda_{n} \in\left(0,1 / \alpha_{i_{n}}\right]$ for all $n \in \mathbb{N}$ and $\liminf _{n \rightarrow \infty} \lambda_{n}\left(1-\alpha_{i_{n}} \lambda_{n}\right)>0$. Then $\left(x_{n}\right)_{n \in \mathbb{N}}$ converges to a point $\bar{x} \in \cap_{i \in I} \operatorname{Fix} T_{i}$.

Proof. For each $i \in I$, by [3, Proposition 4.35], $R_{i}:=\left(1-1 / \alpha_{i}\right) \operatorname{Id}+\left(1 / \alpha_{i}\right) T_{i}$ is nonexpansive. We also have that $\operatorname{Fix} R_{i}=\operatorname{Fix} T_{i}$ and that, for all $n \in \mathbb{N}, x_{n+1}=\left(1-\alpha_{i_{n}} \lambda_{n}\right) x_{n}+\alpha_{i_{n}} \lambda_{n} R_{i_{n}}\left(x_{n}\right)$. Now apply Theorem 4.1 to $\left\{R_{i}\right\}_{i \in I}$ and $\left(\alpha_{i_{n}} \lambda_{n}\right)_{n \in \mathbb{N}}$.

\subsection{Convergence of union nonexpansive iterations}

Using the results of the previous subsection, we now turn our attention convergence of iterations based on union nonexpansive operators. Throughout this subsection, we fix a particular representation for the considered operator. Let $T: X \rightrightarrows X$ denote a union nonexpansive (resp. union $\alpha$-averaged nonexpansive) operator which we assume to be represented as

$$
T(x)=\left\{T_{i}(x): i \in \varphi(x)\right\},
$$

where $I$ is a finite index set, $\left\{T_{i}\right\}_{i \in I}$ is a collection of nonexpansive (resp. $\alpha$-averaged nonexpansive) operators on $X$, and $\varphi: X \rightrightarrows I$ is the osc, nonempty-valued active selector. Fixing this representation is convenient because, in general, the representation of a union nonexpansive (resp. union $\alpha$-averaged nonexpansive) operator need not be unique and allows us to avoid repetition.

Corresponding to the representation (7), we define the radius of attraction of $T$ at a point $x^{*} \in X$ as

$$
r\left(x^{*} ; T\right):=\sup \left\{\delta>0: \forall x \in \mathbb{B}\left(x^{*} ; \delta\right), \varphi(x) \subseteq \varphi\left(x^{*}\right)\right\}
$$

Here we note that radius of attraction is nonzero for a union nonexpansive operator (and hence too for a union averaged nonexpansive operator). In fact, we have that

$$
\forall x^{*} \in X, \quad r\left(x^{*} ; T\right) \in(0,+\infty]
$$

as is shown in the following proposition.

Proposition 4.1. Let $\varphi: X \rightrightarrows I$ for a finite set $I$ and let $x^{*} \in X$. Then $\varphi$ is outer semicontinuous at $x^{*}$ if and only if there exists $\delta>0$ such that

$$
\forall x \in \mathbb{B}\left(x^{*} ; \delta\right), \quad \varphi(x) \subseteq \varphi\left(x^{*}\right) .
$$

Consequently, if $T: X \rightrightarrows X$ is a union nonexpansive operator, then $r\left(x^{*} ; T\right) \in(0,+\infty]$.

Proof. This follows from [5, Proposition 1].

Furthermore, if the reference point $x^{*}$ in Proposition 4.1 is a strong fixed point of the underlying operator $T$, then $T$ satisfies the following quasi-nonexpansiveness properties.

Proposition 4.2 (Radius of attraction at strong fixed points). Let $T: X \rightrightarrows X$ be a union nonexpansive operator with $x^{*} \in \mathbf{F i x} T$. Then $r:=r\left(x^{*} ; T\right) \in(0,+\infty]$ and

$$
\forall x \in \operatorname{int} \mathbb{B}\left(x^{*} ; r\right), \forall y \in T(x), \quad\left\|y-x^{*}\right\| \leq\left\|x-x^{*}\right\| .
$$

Furthermore, for any $\lambda \in(0,1]$, we have

$$
\forall x \in \operatorname{int} \mathbb{B}\left(x^{*} ; r\right), \forall y \in(1-\lambda) x+\lambda T(x), \quad\left\|y-x^{*}\right\|^{2}+\frac{1-\lambda}{\lambda}\|x-y\|^{2} \leq\left\|x-x^{*}\right\|^{2} .
$$

Proof. Since $\varphi$ is osc and its range, $I$, is a finite set, Proposition 4.1 implies that $r:=r\left(x^{*} ; T\right) \in(0,+\infty]$. Since $x^{*} \in \mathbf{F i x} T$, Proposition 3.3(c) implies that $x^{*} \in \cap_{i \in \varphi(x *)} \operatorname{Fix} T_{i}$ and, since each $T_{i}$ is nonexpansive, we have

$$
\forall i \in \varphi\left(x^{*}\right), \forall x \in X, \quad\left\|T_{i}(x)-x^{*}\right\|^{2} \leq\left\|x-x^{*}\right\|^{2} .
$$

In particular, for any $x \in \operatorname{int} \mathbb{B}\left(x^{*} ; r\right)$ and $y \in T(x)$, there exists an $i \in \varphi(x) \subseteq \varphi\left(x^{*}\right)$ such that $y=T_{i}(x)$. Consequently, (9) follows from (11). 
Furthermore, for any $\lambda \in(0,1]$, the operator $S_{i}:=(1-\lambda) \operatorname{Id}+\lambda T_{i}$ is $\lambda$-averaged nonexpansive by Proposition 3.1 and we therefore have that

$$
\forall i \in \varphi\left(x^{*}\right), \forall x \in X, \quad\left\|S_{i}(x)-x^{*}\right\|^{2}+\frac{1-\lambda}{\lambda}\left\|x-S_{i}(x)\right\|^{2} \leq\left\|x-x^{*}\right\|^{2} .
$$

In particular, if $x \in \operatorname{int} \mathbb{B}\left(x^{*} ; r\right)$ and $y \in(1-\lambda) x+\lambda T(x)$, then there exists $i \in \varphi(x) \subseteq \varphi\left(x^{*}\right)$ such that $y=S_{i}(x)$. As before, (10) follows from (12).

We are now ready to prove our main results which establish local convergence of Krasnosel'skiü-Mann iterations based on union nonexpansive and union averaged nonexpansive operators.

Theorem 4.2 (Local convergence of union nonexpansive iterations). Let $T: X \rightrightarrows X$ be a union nonexpansive operator with $x^{*} \in \mathbf{F i x} T$ and let $\left(\lambda_{n}\right)_{n \in \mathbb{N}}$ be a sequence in $(0,1]$ with $\liminf _{n \rightarrow \infty} \lambda_{n}\left(1-\lambda_{n}\right)>0$. Denote $r:=r\left(x^{*} ; T\right) \in(0,+\infty]$ and consider a sequence $\left(x_{n}\right)_{n \in \mathbb{N}}$ with $x_{0} \in \operatorname{int} \mathbb{B}\left(x^{*} ; r\right)$ satisfying

$$
\forall n \in \mathbb{N}, \quad x_{n+1} \in\left(1-\lambda_{n}\right) x_{n}+\lambda_{n} T\left(x_{n}\right) .
$$

Then $\left(x_{n}\right)_{n \in \mathbb{N}}$ converges to a point $\bar{x} \in \operatorname{Fix} T \cap \mathbb{B}\left(x^{*} ; r\right)$.

Proof. We first observe from Proposition 4.2 that, for all $n \in \mathbb{N}, x_{n} \in \operatorname{int} \mathbb{B}\left(x^{*} ; r\right)$ and

$$
\forall n \in \mathbb{N}, \quad\left\|x_{n+1}-x^{*}\right\|^{2}+\frac{1-\lambda_{n}}{\lambda_{n}}\left\|x_{n}-x_{n+1}\right\|^{2} \leq\left\|x_{n}-x^{*}\right\|^{2}
$$

with convention that $\frac{1-\lambda_{n}}{\lambda_{n}}=0$ if $\lambda_{n}=0$. By the definition of $T$, there is a sequence of indices $\left(i_{n}\right)_{n \in \mathbb{N}} \subseteq I$ such that

$$
\forall n \in \mathbb{N}, \quad x_{n+1}=\left(1-\lambda_{n}\right) x_{n}+\lambda_{n} T_{i_{n}}\left(x_{n}\right) \quad \text { and } \quad i_{n} \in \varphi\left(x_{n}\right) \subseteq \varphi\left(x^{*}\right),
$$

where the last inclusion is a consequence of Proposition 4.1 and the fact that $\left(x_{n}\right)_{n \in \mathbb{N}} \subseteq \operatorname{int} \mathbb{B}\left(x^{*} ; r\right)$.

Let $I^{*}$ denote the set of admissible indices in the sequence $\left(i_{n}\right)_{n \in \mathbb{N}}$. Then $I^{*} \subseteq \varphi\left(x^{*}\right)$ which, together with Proposition 3.3(c) applied to $x^{*} \in \mathbf{F i x} T$, yields

$$
x^{*} \in \bigcap_{i \in \varphi(x *)} \operatorname{Fix} T_{i} \subseteq \bigcap_{i \in I^{*}} \operatorname{Fix} T_{i} .
$$

That is, $\left\{T_{i}\right\}_{i \in I^{*}}$ is a collection of nonexpansive operators with a common fixed point. By applying Theorem 4.1, $x_{n} \rightarrow \bar{x} \in \cap_{i \in I^{*}}$ Fix $T_{i}$. Since, for all $n \in \mathbb{N}, x_{n} \in \mathbb{B}\left(x^{*} ; r\right)$, it also holds that $\bar{x} \in \mathbb{B}\left(x^{*} ; r\right)$. Finally, for any $i \in I^{*}$, there exists a subsequence $i_{k_{n}} \rightarrow i$. Since $x_{k_{n}} \rightarrow \bar{x}$ and $\varphi$ is osc with $i_{k_{n}} \in \varphi\left(x_{n_{k}}\right)$, we deduce that $i \in \varphi(\bar{x})$. By Proposition 3.3(b), it follows that $\bar{x} \in \operatorname{Fix} T$ as was claimed.

Corollary 4.2 (Local convergence of union averaged nonexpansive iterations). Let $T: X \rightrightarrows X$ be union $\alpha$-averaged nonexpansive operator with $x^{*} \in \mathbf{F i x} T$ and let $\left(\lambda_{n}\right)_{n \in \mathbb{N}}$ be a sequence in $(0,1 / \alpha]$ with $\liminf _{n \rightarrow \infty} \lambda_{n}\left(1 / \alpha-\lambda_{n}\right)>0$. Denote $r:=r\left(x^{*} ; T\right) \in(0,+\infty]$ and consider $\left(x_{n}\right)_{n \in \mathbb{N}}$ with $x_{0} \in \operatorname{int} \mathbb{B}\left(x^{*} ; r\right)$ satisfying

$$
\forall n \in \mathbb{N}, \quad x_{n+1} \in\left(1-\lambda_{n}\right) x_{n}+\lambda_{n} T\left(x_{n}\right) .
$$

Then $\left(x_{n}\right)_{n \in \mathbb{N}}$ converges to a point $\bar{x} \in \operatorname{Fix} T \cap \mathbb{B}\left(x^{*} ; r\right)$.

Proof. It follows from Proposition 3.1 that $R:=(1-1 / \alpha) \mathrm{Id}+(1 / \alpha) T$ is union nonexpansive. Under the fixed representation of $T$ in (7), we have $r\left(x^{*} ; R\right)=r\left(x^{*} ; T\right)$. Moreover, Fix $R=\mathbf{F i x} T$, Fix $R=$ Fix $T$, and, for all $n \in \mathbb{N}, x_{n+1}=\left(1-\alpha \lambda_{n}\right) x_{n}+\alpha \lambda_{n} R(x)$. The result now follows from Theorem 4.2 applied to $R$ and $\left(\alpha \lambda_{n}\right)_{n \in \mathbb{N}}$.

We conclude this section with the following results concerning global convergence.

Corollary 4.3 (Global convergence of union averaged nonexpansive iterations). Let $T: X \rightrightarrows X$ be union $\alpha$-averaged nonexpansive operator and suppose that there exists $x^{*} \in \mathbf{F i x} T$ with $\varphi\left(x^{*}\right)=I$. Then

$$
\forall x \in X, \forall y \in T(x), \quad\left\|y-x^{*}\right\|^{2}+\frac{1-\alpha}{\alpha}\|x-y\|^{2} \leq\left\|x-x^{*}\right\|^{2} .
$$

Further let $\left(\lambda_{n}\right)_{n \in \mathbb{N}}$ be a sequence in $(0,1 / \alpha]$ with $\liminf _{n \rightarrow \infty} \lambda_{n}\left(1 / \alpha-\lambda_{n}\right)>0$. For any $x_{0} \in X$, consider a sequence $\left(x_{n}\right)_{n \in \mathbb{N}}$ satisfying

$$
\forall n \in \mathbb{N}, \quad x_{n+1} \in\left(1-\lambda_{n}\right) x_{n}+\lambda_{n} T\left(x_{n}\right) .
$$

Then $\left(x_{n}\right)_{n \in \mathbb{N}}$ converges to a point $\bar{x} \in \operatorname{Fix} T$.

Proof. Since $\varphi\left(x^{*}\right)=I$, the radius of attraction (8) at $x^{*}$ is $r\left(x^{*} ; T\right)=+\infty$. Equation (13) now follows from Proposition 4.2 and convergence of $\left(x_{n}\right)_{n \in \mathbb{N}}$ from Corollary 4.2 . 


\subsection{Convergence of iterations based on compositions}

In this subsection, we look at the finer behaviour of the iterates of the compositions of union nonexpansive operators with a common fixed point. As in the previous section, it is convenient to fix representations of said operators. To this end, for each operator $T_{j}$ in the the finite collection of union nonexpansive operators $\left\{T_{j}\right\}_{j \in J}$, we fix the representation

$$
T_{j}(x)=\left\{T_{j, i}(x): i \in \varphi_{j}(x)\right\}
$$

where $I_{j}$ is a finite index set, $\left\{T_{j, i}\right\}_{i \in I_{j}}$ is a collection of nonexpansive operators on $X$, and $\varphi_{j}: X \rightrightarrows I_{j}$ is the osc, nonempty-valued active selector.

Proposition 4.3 (Common fixed points). Let $J:=\{1, \ldots, m\}$ and let $\left\{T_{j}\right\}_{j \in J}$ be a collection of union averaged nonexpansive with the exception of at most one operator which is union nonexpansive. Then for each $x^{*} \in \cap_{i \in J} \mathbf{F i x} T_{j}$, there exists a $\delta>0$ such that

$$
\begin{aligned}
& \operatorname{Fix}\left(T_{m} \circ \cdots \circ T_{1}\right) \cap \mathbb{B}\left(x^{*} ; \delta\right)=\bigcap_{j \in J} \operatorname{Fix} T_{j} \cap \mathbb{B}\left(x^{*} ; \delta\right), \\
& \operatorname{Fix}\left(T_{m} \circ \cdots \circ T_{1}\right) \cap \mathbb{B}\left(x^{*} ; \delta\right)=\bigcap_{j \in J} \operatorname{Fix} T_{j} \cap \mathbb{B}\left(x^{*} ; \delta\right) .
\end{aligned}
$$

Proof. We first observe that $\cap_{j \in J} \mathbf{F i x} T_{j} \subseteq \operatorname{Fix}\left(T_{m} \circ \cdots \circ T_{1}\right)$ and $\cap_{j \in J} \operatorname{Fix} T_{j} \subseteq \operatorname{Fix}\left(T_{m} \circ \cdots \circ T_{1}\right)$. Thus to prove the claimed result, we need only establish that for each $m$ there exists a $\delta>0$ such that

$$
\begin{aligned}
& \operatorname{Fix}\left(T_{m} \circ \cdots \circ T_{1}\right) \cap \mathbb{B}\left(x^{*} ; \delta\right) \subseteq \bigcap_{j \in J} \operatorname{Fix} T_{j}, \\
& \operatorname{Fix}\left(T_{m} \circ \cdots \circ T_{1}\right) \cap \mathbb{B}\left(x^{*} ; \delta\right) \subseteq \bigcap_{j \in J} \operatorname{Fix} T_{j} .
\end{aligned}
$$

To do so, we use induction on $m$. First, it is clear that (14) holds for $m=1$, so there is nothing to do. Suppose instead that (14) $m \geq 2$ and the result holds for $1,2, \ldots, m-1$. Combining the assumptions on $\left\{T_{i}\right\}_{i \in I}$ with Proposition 3.2(c), we deduce the existence of an index $k \in J \backslash\{m\}$ such that both $S_{2}:=T_{m} \circ \cdots \circ T_{k+1}$ and $S_{1}:=T_{k} \circ \cdots \circ T_{1}$ are union nonexpansive, and at least one of $S_{1}$ or $S_{2}$ is union averaged nonexpansive. Then

$$
x^{*} \in \bigcap_{i \in J} \mathbf{F i x} T_{j}=\left(\bigcap_{i=1}^{k} \mathbf{F i x} T_{j}\right) \cap\left(\bigcap_{j=k+1}^{m} \mathbf{F i x} T_{j}\right) \subseteq \mathbf{F i x} S_{1} \cap \mathbf{F i x} S_{2} .
$$

By the induction hypothesis, there exists a $\delta>0$ such that

$$
\begin{array}{ll}
\operatorname{Fix}\left(S_{1}\right) \cap \mathbb{B}\left(x^{*} ; \delta\right) \subseteq \bigcap_{j=1}^{k} \operatorname{Fix} T_{j}, & \boldsymbol{F i x}\left(S_{2}\right) \cap \mathbb{B}\left(x^{*} ; \delta\right) \subseteq \bigcap_{j=k+1}^{m} \operatorname{Fix} T_{j}, \\
\operatorname{Fix}\left(S_{1}\right) \cap \mathbb{B}\left(x^{*} ; \delta\right) \subseteq \bigcap_{j=1}^{k} \operatorname{Fix} T_{j}, & \operatorname{Fix}\left(S_{2}\right) \cap \mathbb{B}\left(x^{*} ; \delta\right) \subseteq \bigcap_{j=k+1}^{m} \operatorname{Fix} T_{j} .
\end{array}
$$

By shrinking $\delta$ if necessary, we may and do assume that $0<\delta<\min \left\{r\left(x^{*} ; S_{1}\right), r\left(x^{*} ; S_{2}\right)\right\}$. Now, let $x \in \mathbf{F i x}\left(S_{2} \circ S_{1}\right) \cap \mathbb{B}\left(x^{*} ; \delta\right)$. Then $\{x\}=S_{2}(y)$ for all $y \in S_{1}(x)$. Since $x^{*} \in \mathbf{F i x} S_{1} \cap \mathbf{F i x} S_{2}$, and either $S_{1}$ or $S_{2}$ is union $\alpha$-averaged nonexpansive, Proposition 4.2 applied to $S_{1}$ and then $S_{2}$ yields

$$
\forall y \in S_{1}(x), \quad\left\|x-x^{*}\right\|^{2} \geq\left\|x-x^{*}\right\|^{2}+\frac{1-\alpha}{\alpha}\|x-y\|^{2} .
$$

Hence we have that $\|x-y\|=0$ for all $y \in S_{1}(x)$ or, equivalently, that $S_{1}(x)=\{x\}$. It then follows that $x \in \mathbf{F i x} S_{1} \cap \mathbf{F i x} S_{2} \cap \mathbb{B}\left(x^{*} ; \delta\right)$ which proves the equality for the strong fixed point set. The proof for the fixed point set is performed similarly.

In order to prove the main result of this subsection, we require the following technical lemma. 
Lemma 4.1. Let $\left\{T_{j}\right\}_{j \in J}$ be a finite collection of union nonexpansive operators on $X$ with $x^{*} \in$ $\cap_{j \in J} \mathbf{F i x} T_{j}$ and let $\left(\lambda_{n}\right)_{n \in \mathbb{N}}$ be a sequence in $\left.] 0,1\right]$ with $\lim \inf _{n \rightarrow \infty} \lambda_{n}\left(1-\lambda_{n}\right)>0$. Denote

$$
r:=\min _{j \in J} r\left(x^{*} ; T_{j}\right) \in(0,+\infty]
$$

and consider a sequence $\left(x_{n}\right)_{n \in \mathbb{N}}$ with $x_{0} \in \operatorname{int} \mathbb{B}\left(x^{*} ; r\right)$ satisfying

$$
\forall n \in \mathbb{N}, \quad x_{n+1} \in\left(1-\lambda_{n}\right) x_{n}+\lambda_{n} T_{j_{n}}\left(x_{n}\right),
$$

where $\left(j_{n}\right)_{n \in \mathbb{N}}$ is admissible in $J$. Then the sequence $\left(x_{n}\right)_{n \in \mathbb{N}}$ converges and its limit is contained in $\left(\cup_{j \in J} \operatorname{Fix} T_{j}\right) \cap \mathbb{B}\left(x^{*} ; r\right)$.

Proof. Set $T:=\cup_{j \in J} T_{j}$. By Proposition 3.2(a), $T$ is a union nonexpansive operator with active selector

$$
\varphi(x):=\left\{(j, i): j \in J, i \in \varphi_{j}(x)\right\} .
$$

For any $x \in X$, we have $\varphi(x) \subseteq \varphi\left(x^{*}\right)$ if and only if $\varphi_{j}(x) \subseteq \varphi_{j}\left(x^{*}\right)$ for all $j \in J$. It thus follows that

$$
r\left(x^{*} ; T\right)=\min _{j \in J} r\left(x^{*} ; T_{j}\right) \in(0,+\infty]
$$

where positivity of the right-hand-side follows from Proposition 4.1 and the finiteness of $J$. A direct calculation then shows that

$$
\operatorname{Fix} T=\bigcap_{j \in J} \operatorname{Fix} T_{j} \quad \text { and } \quad \operatorname{Fix} T=\bigcup_{j \in J} \operatorname{Fix} T_{j} .
$$

We also note that every sequence generated by (15) is actually a sequence with the same starting point generated by

$$
\forall n \in \mathbb{N}, \quad x_{n+1} \in\left(1-\lambda_{n}\right) x_{n}+\lambda_{n} T\left(x_{n}\right) .
$$

The result thus follows from Theorem 4.2 applied to $T$.

In the following corollary, $n \bmod m \in\{0, \ldots, m-1\}$ denotes the remainder when $n$ is divided by $m$.

Corollary 4.4 (Local convergence of compositions). Let $J:=\{1, \ldots, m\}$ and let $\left\{T_{j}\right\}_{j \in J}$ be a collection of union averaged nonexpansive operators on $X$ with $x^{*} \in \mathbf{F i x}\left(T_{m} \circ \cdots \circ T_{1}\right)$. Denote $r \in$ $\left(0, r\left(x^{*} ; T_{m} \circ \cdots \circ T_{1}\right)\right]$ and consider a sequence $\left(x_{n}\right)_{n \in \mathbb{N}}$ with $x_{0} \in \operatorname{int} \mathbb{B}\left(x^{*} ; r\right)$ satisfying

$$
\forall n \in \mathbb{N}, \quad x_{n+1} \in T_{i_{n}}\left(x_{n}\right), \text { where } i_{n}=(n \bmod m)+1 .
$$

Then $\left(x_{m n}\right)_{n \in \mathbb{N}}$ converges to a point $\bar{x} \in \operatorname{Fix}\left(T_{m} \circ \cdots \circ T_{1}\right) \cap \mathbb{B}\left(x^{*} ; r\right)$. Furthermore, if $x^{*} \in \cap_{j \in J} \mathbf{F i x} T_{j}$ and $r$ is sufficiently small, then the entire sequence $\left(x_{n}\right)_{n \in \mathbb{N}}$ converges to $\bar{x}$ and $\bar{x} \in \cap_{j \in J} \operatorname{Fix} T_{j} \cap \mathbb{B}\left(x^{*} ; r\right)$.

Proof. Since $T:=T_{m} \circ \cdots \circ T_{1}$ is union averaged nonexpansive by Proposition 3.2(c), the claim regarding convergence of $\left(x_{m n}\right)_{n \in \mathbb{N}}$ to a point $\bar{x} \in \operatorname{Fix}\left(T_{m} \circ \cdots \circ T_{1}\right)$ follows by applying Corollary 4.2 to $T$ with all $\lambda_{n}=1$. To prove the second claim, first note that the entire sequence $\left(x_{n}\right)_{n \in \mathbb{N}}$ is convergent by Lemma 4.1. Since one of its subsequence, $\left(x_{m n}\right)_{n \in \mathbb{N}}$, converges to $\bar{x}$, it must be that $x_{n} \rightarrow \bar{x}$. Now, Proposition 4.3 completes the proof.

\section{Min-convex functions}

In this section we study the following class of functions whose proximity operators will be shown to belong to the class of union averaged nonexpansive operators.

Definition 5.1 (Min-convexity). We say a function $f: X \rightarrow(-\infty,+\infty]$ is min-convex if it can be expressed in the form

$$
\forall x \in X, \quad f(x):=\min _{i \in I} f_{i}(x),
$$

where $I$ is a finite index set and the functions $f_{i}: X \rightarrow(-\infty,+\infty]$ are proper, lsc and convex.

In general, a min-convex function need not be convex. In fact, sufficient conditions for a min-convex function to be convex were studied in [10] (see also [11, Proposition 5]). As a concrete example of a min-convex function, we revisit Example 3.1. 
Example 5.1 (Sparsity projectors (revisited)). Let $X=\mathbb{R}^{n}$ and $s \in\{0,1, \ldots, n-1\}$. Recall that the sparsity constraint from Example 3.1 which can be expresses as the union of subspaces. i.e.,

$$
C:=\left\{x \in \mathbb{R}^{n}:\|x\|_{0} \leq s\right\}=\bigcup_{I \in \mathcal{I}} C_{I} \text { where } C_{I}:=\left\{x \in \mathbb{R}^{n}: x_{i} \neq 0 \text { only if } i \in I\right\}
$$

Due to this representation, we see that the indicator function to $C, \iota_{C}$, can be expressed as $\iota_{C}=$ $\min _{I \in \mathcal{I}} \iota_{C_{I}}$. As the indicator function to a (closed) subspace, is a proper lsc convex function, we see that $\iota_{C}$ is min-convex.

In the subsequent section, we shall study proximal algorithms for min-convex functions. These algorithms are based upon the following two objects.

Definition 5.2 (Moreau envelopes and proximity operators). Let $f: X \rightarrow(-\infty,+\infty]$ be a proper function and let $\gamma>0$ be a positive parameter. The Moreau envelope of $f$ denoted ${ }^{\gamma} f: X \rightarrow(-\infty,+\infty]$ is the function

$$
\gamma_{f}(x):=\inf _{y \in X}\left(f(y)+\frac{1}{2 \gamma}\|x-y\|^{2}\right)
$$

and the proximity operator of $f$ denoted $\operatorname{prox}_{\gamma f}: X \rightrightarrows X$ is given by

$$
\operatorname{prox}_{\gamma f}(x)=\left\{y \in X: f(y)+\frac{1}{2 \gamma}\|x-y\|^{2}=\gamma_{f}(x)\right\} \text {. }
$$

It is well known that when $f$ is proper, lsc and convex, its proximity operator is single-valued and firmly nonexpansive (i.e., 1/2-averaged nonexpansive) (see, for instance, [3, Proposition 12.28]).

Recall that the proximal subdifferential of $f: X \rightarrow(-\infty,+\infty]$ at $x \in X$ is given by

$$
\partial_{p} f(x):=\left\{x^{*} \in X: \exists \gamma, \delta>0, \forall y \in \mathbb{B}(x ; \delta),\left\langle x^{*}, y-x\right\rangle \leq f(y)-f(x)+\frac{1}{2 \gamma}\|y-x\|^{2}\right\}
$$

and that $0 \in \partial_{p} f(x)$ whenever $x$ is a local minimum of $f$; see, e.g., [12, Equations (0.1) and (1.4)].

In the following two propositions, we investigate various properties under assumptions which are satisfied by min-convex functions.

Proposition 5.1 (Properties of proper functions). Let $f: X \rightarrow(-\infty,+\infty]$ be proper and let $\gamma>0$. The following assertions hold.

(a) $\operatorname{dom}^{\gamma} f=X$.

(b) $\forall x \in X$, inf $f(X)=\inf ^{\gamma} f(X) \leq \gamma f(x) \leq f(x)$.

(c) Let $x, p \in X$. Then $p \in \operatorname{prox}_{\gamma f}(x)$ if and only if

$$
\forall y \in X, \quad\left\langle\frac{1}{\gamma}(x-p), y-p\right\rangle \leq f(y)-f(p)+\frac{1}{2 \gamma}\|y-p\|^{2} .
$$

In particular, if $p \in \operatorname{prox}_{\gamma f}(x)$, then $\frac{1}{\gamma}(x-p) \in \partial_{p} f(p)$.

(d) Fix $\operatorname{prox}_{\gamma f}=\left\{x \in X:{ }^{\gamma} f(x)=f(x)\right\}$.

(e) The following inclusions hold.

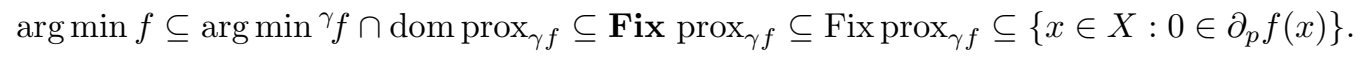

Moreover, when $f$ is convex, all inclusions are satisfied with equality.

Proof. (a) \& (b): See, for instance, [3, Proposition 12.9(i)-(iii)].

(c): Using definition of the $\operatorname{prox}_{\gamma f}$, we deduce that

$$
\begin{aligned}
p \in \operatorname{prox}_{\gamma f}(x) & \Longleftrightarrow \forall y \in X, \quad f(p)+\frac{1}{2 \gamma}\|x-p\|^{2} \leq f(y)+\frac{1}{2 \gamma}\|x-y\|^{2} \\
& \Longleftrightarrow \forall y \in X, \quad\left\langle\frac{1}{\gamma}(x-p), y-p\right\rangle \leq f(y)-f(p)+\frac{1}{2 \gamma}\|y-p\|^{2}
\end{aligned}
$$




$$
\Longrightarrow \frac{1}{\gamma}(x-p) \in \partial_{p} f(p)
$$

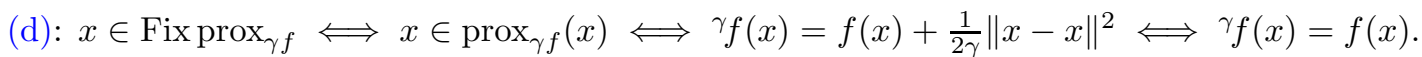

(e): To show the first inclusion, let $x \in \arg \min f$. By applying (b), we deduce that $f(x)=\inf f(X)=$ $\inf ^{\gamma} f(X) \leq{ }^{\gamma} f(x) \leq f(x)$, and so ${ }^{\gamma} f(x)=\inf ^{\gamma} f(X)=f(x)=f(x)+\frac{1}{2 \gamma}\|x-x\|^{2}$, which yields $x \in$ $\arg \min { }^{\gamma} f \cap \operatorname{dom}_{\operatorname{prox}}$. We therefore obtain that $\arg \min f \subseteq \arg \min ^{\gamma} f \cap \operatorname{dom} \operatorname{prox}_{\gamma f}$.

To prove the second inclusion, let $x \in \arg \min ^{\gamma} f \cap \operatorname{dom}_{\operatorname{prox}}$. Then there exists $p \in \operatorname{prox}_{\gamma f}(x)$ and using (b) we deduce that

$$
\inf ^{\gamma} f(X) \leq f(p) \leq{ }^{\gamma} f(x)=\min ^{\gamma} f(X),
$$

which implies that $f(p)=\gamma_{f}(x)=f(p)+\frac{1}{2 \gamma}\|x-p\|^{2}$, hence we conclude that $p=x$. In other words,

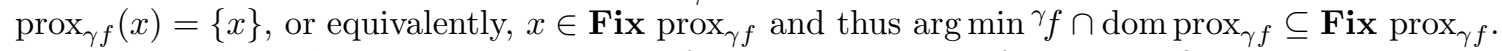

The inclusion Fix $\operatorname{prox}_{\gamma f} \subseteq$ Fix $\operatorname{prox}_{\gamma f}$ follows immediately from the definition. To prove the final inclusion, let $x \in$ Fix $\operatorname{prox}_{\gamma f}$. Then $x \in \operatorname{prox}_{\gamma f}(x)$ and, by (c), $0 \in \partial_{p} f(x)$ as was claimed. In the case in which $f$ is convex, by [13, Proposition 7.26] we have $\partial_{p} f=\partial f$, and by [3, Theorem 16.3] we have

$$
\left\{x \in X: 0 \in \partial_{p} f(x)\right\}=\{x \in X: 0 \in \partial f(x)\}=\arg \min f,
$$

from which the claimed equalities follow.

Proposition 5.2 (Properties of min-convex functions). Let I be a finite index set, let $f=\min _{i \in I} f_{i}$ with $f_{i}: X \rightarrow(-\infty,+\infty]$ proper, and let $\gamma>0$. The following assertions hold.

(a) $\forall x \in X,{ }^{\gamma} f(x)=\min _{i \in I}{ }^{\gamma} f_{i}(x)$.

(b) $\forall x \in X, \operatorname{prox}_{\gamma f}(x)=\left\{\operatorname{prox}_{\gamma f_{i}}(x): i \in I, \gamma_{f}(x)={ }^{\gamma} f_{i}(x)\right\}$.

(c) Fix $\operatorname{prox}_{\gamma f} \subseteq\left\{x \in X: 0 \in \partial_{p} f_{i}(x)\right.$ whenever $\left.f(x)=f_{i}(x)\right\}$.

Further suppose that $f$ is min-convex (i.e., $f_{i}$ is lsc and convex, for each $i \in I$ ). Then

(d) $\{x \in X: x$ is a local minimum of $f\}=\left\{x \in X: x \in \arg \min f_{i}\right.$ whenever $\left.f(x)=f_{i}(x)\right\}$.

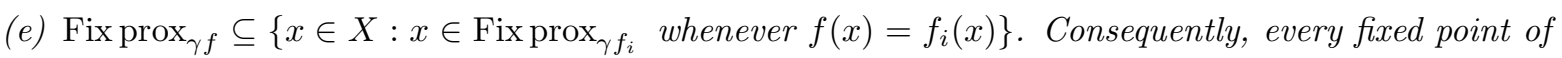
$\operatorname{prox}_{\gamma f}$ is a local minimum of $f$.

(f) $\operatorname{prox}_{\gamma f}$ is union $1 / 2$-averaged nonexpansive. In particular, $\operatorname{prox}_{\gamma f}$ can be expressed as

$$
\operatorname{prox}_{\gamma f}(x)=\left\{\operatorname{prox}_{\gamma f_{i}}(x): i \in \varphi(x)\right\}
$$

with active selector $\varphi: X \rightrightarrows I$ given by $\varphi(x)=\left\{i \in I:{ }^{\gamma} f(x)={ }^{\gamma} f_{i}(x)\right\}$.

Proof. (a): For all $x \in X$, we have that

$$
\begin{aligned}
\gamma_{f(x)} & =\inf _{y \in X}\left(\min _{i \in I} f_{i}(y)+\frac{1}{2 \gamma}\|x-y\|^{2}\right) \\
& =\inf _{y \in X} \min _{i \in I}\left(f_{i}(y)+\frac{1}{2 \gamma}\|x-y\|^{2}\right) \\
& =\min _{i \in I} \inf _{y \in X}\left(f_{i}(y)+\frac{1}{2 \gamma}\|x-y\|^{2}\right)=\min _{i \in I}^{\gamma} f_{i}(x),
\end{aligned}
$$

where interchanging infimum and minimum is valid due to the finiteness of $I$.

(b): Suppose $p \in \operatorname{prox}_{\gamma f}(x)$. Since $I$ is finite, there exists an index $i \in I$ such that $f(p)=f_{i}(p)$. Consequently, we have

$$
{ }^{\gamma} f(x)=f(p)+\frac{1}{2 \gamma}\|x-p\|^{2}=f_{i}(p)+\frac{1}{2 \gamma}\|x-p\|^{2} \geq{ }^{\gamma} f_{i}(x) .
$$

Together with (a), this implies that ${ }^{\gamma} f(x)={ }^{\gamma} f_{i}(x)$ and that $p \in \operatorname{prox}_{\gamma f_{i}}(x)$.

To prove the reverse inclusion, suppose $p \in \operatorname{prox}_{\gamma f_{i}}(x)$ for some $i \in I$ such that ${ }^{\gamma} f(x)={ }^{\gamma} f_{i}(x)$. Then

$$
{ }^{\gamma} f(x)={ }^{\gamma} f_{i}(x)=f_{i}(p)+\frac{1}{2 \gamma}\|x-p\|^{2} \geq f(p)+\frac{1}{2 \gamma}\|x-p\|^{2} \geq{ }^{\gamma} f(x),
$$


which implies that $p \in \operatorname{prox}_{\gamma f}(x)$ and thus completes the proof of (b).

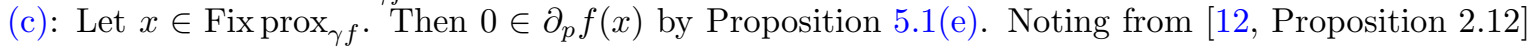
that

$$
\partial_{p} f(x) \subseteq \bigcap_{i \in I, f(x)=f_{i}(x)} \partial_{p} f_{i}(x) .
$$

we have $0 \in \partial_{p} f_{i}(x)$ whenever $f(x)=f_{i}(x)$. The claim follows.

(d): First note that, for every $i \in I$, the convexity of $f_{i}$ combined with Proposition 5.1(e) implies that

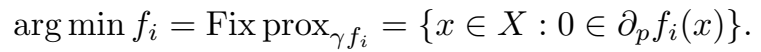

Now, let $x$ be a local minimum of $f$. Then $0 \in \partial_{p} f(x)$ and, by (18), $0 \in \partial_{p} f_{i}(x)$ whenever $f(x)=f_{i}(x)$. Together with (19), this yields $x \in \arg \min f_{i}$ whenever $f(x)=f_{i}(x)$.

Conversely, consider a point $x$ such that $x \in \arg \min f_{i}$ whenever $f(x)=f_{i}(x)$. Suppose, by way of a contradiction, that $x$ is not a local minimum of $f$. Then there exists a sequence $\left(y_{n}\right)_{n \in \mathbb{N}}$ such that $y_{n} \in \mathbb{B}(x ; 1 / n)$ and $f\left(y_{n}\right)<f(x)$. Set $I_{0}:=\left\{i \in I: f(x)=f_{i}(x)\right\}$. Then

$$
\forall i \in I_{0}, \forall n \in \mathbb{N}, \quad f\left(y_{n}\right)<f(x)=f_{i}(x) \leq f_{i}\left(y_{n}\right),
$$

where the last inequality holds because $x \in \arg \min f_{i}$. Therefore, for each $n \in \mathbb{N}$, there exits a $j_{n} \in I \backslash I_{0}$ such that $f\left(y_{n}\right)=f_{j_{n}}\left(y_{n}\right)$. As $I$ is finite, by passing to a subsequence if necessary, we can and do assume that there is $j \in I \backslash I_{0}$ such that $f\left(y_{n}\right)=f_{j}\left(y_{n}\right)$ for all $n \in \mathbb{N}$. Noting that $y_{n} \rightarrow x$ and using lower semicontinuity of $f_{j}$ give

$$
f_{j}(x) \leq \liminf _{n \rightarrow \infty} f_{j}\left(y_{n}\right)=\liminf _{n \rightarrow \infty} f\left(y_{n}\right) \leq f(x)=\min _{i \in I} f_{i}(x),
$$

which implies that $j \in I_{0}$; a contradiction.

(e): Combine (c), (d), and (19).

(f): Using (b), we have that $\operatorname{prox}_{\gamma f}(x)=\left\{\operatorname{prox}_{\gamma f_{i}}(x): i \in \varphi(x)\right\}$ with $\varphi(x)=\left\{i \in I:{ }^{\gamma} f(x)={ }^{\gamma} f_{i}(x)\right\}$ for all $x \in X$. By [3, Proposition 12.28], $\operatorname{prox}_{\gamma f_{i}}$ is $1 / 2$-averaged nonexpansive for each $i \in I$, hence only osc of the active selector $\varphi$ remains to be verified. To this end, consider sequences $\left(x_{n}, i_{n}\right) \rightarrow(x, i)$ with $i_{n} \in \varphi\left(x_{n}\right)$ for all $n \in \mathbb{N}$. Because $I$ is finite, by passing to a subsequence, we may assume that $i_{n}=i$ for all $n \in \mathbb{N}$. Since ${ }^{\gamma} f_{j}$ is continuous for each $j \in J$ [3, Proposition 12.15], we have

$$
{ }^{\gamma} f_{i}(x)=\lim _{n \rightarrow \infty}{ }^{\gamma} f\left(x_{n}\right)=\lim _{n \rightarrow \infty} \min _{j \in I}{ }^{\gamma} f_{j}\left(x_{n}\right)=\min _{j \in I} \lim _{n \rightarrow \infty}{ }^{\gamma} f_{j}\left(x_{n}\right)=\min _{j \in I}{ }^{\gamma} f_{j}(x)={ }^{\gamma} f(x) .
$$

This shows that $i \in \varphi(x)$ and completes the proof.

To conclude this section, we introduce one further notion which we shall require in certain cases of our analysis. It can be viewed as a kind of constraint qualification on the representation of a min-convex function.

Definition 5.3 (Outer semicontinuous representations). Let $f: X \rightarrow(-\infty,+\infty]$ be a min-convex function. We say $f$ is outer semicontinuously (osc) representable at $\bar{x} \in X$ if there exists a min-convex representation, $f=\min _{i \in I} f_{i}$, such that the selector $\phi: X \rightrightarrows I$ is osc at $\bar{x}$ where

$$
\phi(x):=\left\{i \in I: f(x)=f_{i}(x)\right\} .
$$

If there exists a single representation such that $\phi$ is everywhere osc, then we say that $f$ is osc representable.

Clearly every convex function is osc representable. Moreover, the following proposition shows that, in particular, the Moreau envelope of a min-convex function is also osc representable.

Proposition 5.3. Let $I$ be a finite index set, let $f_{i}: X \rightarrow \mathbb{R}$ be continuous, and set $f:=\min _{i \in I} f_{i}$. Then the selector $\phi(x):=\left\{i \in I: f(x)=f_{i}(x)\right\}$ is osc. Consequently, the Moreau envelope of a min-convex function is always osc representable.

Proof. Let $x_{n} \rightarrow x$ and $i_{n} \rightarrow i$ with $i_{n} \in \phi\left(x_{n}\right)$ for all $n \in \mathbb{N}$. Since $I$ is finite, there exists an $n_{0} \in \mathbb{N}$ such that $i_{n}=i$ for all $n \geq n_{0}$. Since $\left\{f_{i}\right\}$ are continuous, $f$ is also continuous as the minimum of continuous functions. Consequently,

$$
f_{i}(x)=\lim _{n \rightarrow \infty} f_{i}\left(x_{n}\right)=\lim _{n \rightarrow \infty} f\left(x_{n}\right)=f(x),
$$

which shows that $i \in \phi(x)$ and establishes the osc of $\phi$.

Now, if a function $g: X \rightarrow(-\infty,+\infty]$ is min-convex, there exists a finite index set, $I$, and proper, lsc convex functions $\left\{g_{i}\right\}_{i \in I}$ such that $g=\min _{i \in I} g_{i}$. For any $\gamma>0$, Proposition 5.2(a) shows that ${ }^{\gamma} g=\min _{i \in I}{ }^{\gamma} g_{i}$. Since the Moreau envelope of a proper, lsc convex function is always continuous [3, Proposition 12.15], the claim follows. 


\section{Proximal algorithms for min-convex minimization}

In this section, we use the results of the last two sections to systematically analyze proximal algorithms applied to min-convex functions. We consider four different settings: projection algorithms, the proximal point algorithm, the forward-backward method, and Douglas-Rachford splitting.

\subsection{Projection algorithms}

Given sets $C_{1}, \ldots, C_{m} \subseteq X$ with nonempty intersection, the feasibility problem is to

$$
\text { find } x \in \bigcap_{j=1}^{m} C_{j} \text {. }
$$

In this section, we consider the case in which each set $C_{j}$ is union convex by which we mean that it can be expressed as a finite union of closed convex sets. Recall that the projector onto a nonempty set $C$ in $X$ is the set-valued operator $P_{C}: X \rightrightarrows C$ define by

$$
P_{C}(x):=\operatorname{prox}_{\iota_{C}}(x)=\underset{c \in C}{\arg \min }\|x-c\|=\{c \in C:\|x-c\|=\mathrm{d}(x, C)\},
$$

where $\mathrm{d}(x, C):=\inf _{c \in C}\|x-c\|$ is the distance from $x$ to $C$.

Proposition 6.1 (Union convex sets). Let $A=\cup_{i \in I} A_{i}$ and $B=\cup_{j \in J} B_{j}$ where $I, J$ are finite index sets and $A_{i}, B_{j}$ are nonempty closed convex sets in $X$. The following assertions hold.

(a) The projector $P_{A}$ is union 1/2-averaged nonexpansive with

$$
P_{A}(x)=\left\{P_{A_{i}}(x): i \in I, \mathrm{~d}\left(x, A_{i}\right)=\mathrm{d}(x, A)\right\}
$$

and the reflector $R_{A}:=2 P_{A}-\mathrm{Id}$ is union nonexpansive.

(b) The Douglas-Rachford (DR) operator given by

$$
T_{A, B}(x):=\frac{\operatorname{Id}+R_{B} \circ R_{A}}{2}(x)=\left\{x+b-a \in X: a \in P_{A}(x), b \in P_{B}(2 a-x)\right\}
$$

is union 1/2-averaged nonexpansive.

Proof. (a): Let $f_{i}=\iota_{A_{i}}$ in Proposition 5.2(f) and then apply Proposition 3.1.

(b): Since both $R_{A}$ and $R_{B}$ are union nonexpansive by (a), Proposition 3.2(c) implies that $R_{B} R_{A}$ is also union nonexpansive. The result then follows by applying Proposition $3.1(\mathrm{~b})$ to $R_{B} R_{A}$ with $\alpha=1 / 2$.

Remark 6.1. In the setting of Proposition 6.1, one can also deduce union averaged nonexpansiveness of relaxations of projection operators such as those consider in the so-called generalized Douglas-Rachford operator $[14,15]$ which includes the relaxed averaged alternating reflection operator [16]. However, we shall focus on algorithms involving projectors and (ungeneralized) Douglas-Rachford operators.

We now state our results regarding convergence of projection algorithms. We consider three different algorithms: the method of cyclic projections, the cyclic Douglas-Rachford method [17, 18] and the cyclically anchored Douglas-Rachford method [19]. The latter includes the usual two-set Douglas-Rachford method as a special case.

Theorem 6.1 (Projection algorithms on union convex sets). Let $J:=\{1, \ldots, m\}$ and let $\left\{C_{j}\right\}_{j \in J}$ be a finite collection of union convex sets in $X$. Given $x_{0} \in X$, define $x_{n+1} \in T\left(x_{n}\right)$ for all $n \in \mathbb{N}$ in any one of the following cases.

(a) (method of cyclic projections) $T=P_{C_{m}} \circ \cdots \circ P_{C_{2}} \circ P_{C_{1}}$.

(b) (cyclic Douglas-Rachford method) $T=T_{C_{m}, C_{1}} \circ \cdots \circ T_{C_{2}, C_{3}} \circ T_{C_{1}, C_{2}}$.

(c) (cyclically anchored Douglas-Rachford method) $T=T_{C_{1}, C_{m}} \circ \cdots \circ T_{C_{1}, C_{3}} \circ T_{C_{1}, C_{2}}$.

Then $\cap_{j \in J} C_{j} \subseteq \mathbf{F i x} T$. Moreover, if $x^{*} \in \mathbf{F i x} T$, then there exists $r>0$ such that, whenever $x_{0} \in$ int $\mathbb{B}\left(x^{*} ; r\right)$, the sequence $\left(x_{n}\right)_{n \in \mathbb{N}}$ converges to a point $\bar{x} \in \operatorname{Fix} T \cap \mathbb{B}\left(x^{*} ; r\right)$. 
Proof. It is straightforward to check that $\cap_{j \in J} C_{j} \subseteq \mathbf{F i x} T$. To show the second claim, first combine Proposition 3.2(c) and Proposition 6.1 to deduce that $T$ is union averaged nonexpansive. The results then follows from Corollary 4.2 with $\lambda_{n}=1$.

Theorem 6.2 (Cyclically anchored Douglas-Rachford method). Let $J:=\{1, \ldots, m\}$ and suppose $\left\{C_{j}\right\}_{j \in J}$ is a finite collection of union convex sets in $X$. Consider a sequence $\left(x_{n}\right)_{n \in \mathbb{N}}$ with $x_{0} \in X$ satisfying

$$
\forall n \in \mathbb{N}, \quad x_{n+1} \in T_{C_{1}, C_{i_{n}}}\left(x_{n}\right) \text { where } i_{n}=(n \bmod (m-1))+2 .
$$

If $x^{*} \in \cap_{j \in J \backslash\{1\}} \mathbf{F i x} T_{C_{1}, C_{j}}$ (in particular, if $x^{*} \in \cap_{i \in J} C_{j}$ ), then there exists an $r>0$ such that the sequence $\left(x_{n}\right)_{n \in \mathbb{N}}$ converges to a point $\bar{x} \in \cap_{j \in J \backslash\{1\}}$ Fix $T_{C_{1}, C_{j}}$ whenever $x_{0} \in \operatorname{int} \mathbb{B}\left(x^{*} ; r\right)$. Moreover, if the set $C_{1}$ is convex, then $P_{C_{1}}(\bar{x}) \in \cap_{j \in J} C_{j}$.

Proof. Let $x^{*} \in \cap_{j \in J \backslash\{1\}} \mathbf{F i x} T_{C_{1}, C_{j}}$. For every $j \in J \backslash\{1\}$, the definition of $T_{C_{1}, C_{j}}$ yields $C_{1} \cap C_{j} \subseteq$ Fix $T_{C_{1}, C_{j}}$. Consequently, we have

$$
\cap_{j \in J} C_{j} \subseteq \cap_{j \in J \backslash\{1\}} \mathbf{F i x} T_{C_{1}, C_{j}} \subseteq \mathbf{F i x}\left(T_{C_{1}, C_{m}} \circ \cdots \circ T_{C_{1}, C_{2}}\right),
$$

which shows, in particular, that $x^{*}$ is strong fixed point of the cyclically anchored Douglas-Rachford operator. By Proposition 6.1(b), $\left\{T_{C_{1}, C_{j}}\right\}_{j \in J \backslash\{1\}}$ is a collection of union $1 / 2$-averaged operators. By setting $r:=\min _{j \in J \backslash\{1\}} r\left(x^{*} ; T_{C_{1}, C_{j}}\right)>0$ and applying Corollary 4.4, we deduce that the sequence $\left(x_{n}\right)_{n \in \mathbb{N}}$ converges to a point $\bar{x} \in \cap_{j \in J \backslash\{1\}}$ Fix $T_{C_{1}, C_{j}}$ whenever $x_{0} \in \operatorname{int} \mathbb{B}\left(x^{*} ; r\right)$, which proves the first claim. Moreover, if $C_{1}$ is convex, then [20, Equation (23)] implies that $P_{C_{1}}(\bar{x}) \in C_{1} \cap C_{j}$ for every $j \in J \backslash\{1\}$, which completes the proof.

In particular, setting $m=2$ in Theorem 6.2, we recover the result for the usual two-set DouglasRachford method as a special case. This was original proven by Bauschke and Noll [6, Theorem 1].

Corollary 6.1 (Douglas-Rachford method [6, Theorem 1]). Let $C_{1}$ and $C_{2}$ be union convex sets in $X$. Consider a sequence $\left(x_{n}\right)_{n \in \mathbb{N}}$ with $x_{0} \in X$ satisfying

$$
\forall n \in \mathbb{N}, \quad x_{n+1} \in T_{C_{1}, C_{2}}\left(x_{n}\right) .
$$

If $x^{*} \in \mathbf{F i x} T_{C_{1}, C_{2}}$ (in particular, if $x^{*} \in C_{1} \cap C_{2}$ ), then there exists an $r>0$ such that the sequence $\left(x_{n}\right)_{n \in \mathbb{N}}$ converges to a point $\bar{x} \in \operatorname{Fix} T_{C_{1}, C_{2}}$ whenever $x_{0} \in \operatorname{int} \mathbb{B}\left(x^{*} ; r\right)$. Moreover, there exists a point $c \in P_{C_{1}}(\bar{x})$ such that $c \in C_{1} \cap C_{2}$.

Proof. By applying Theorem 6.2 with $m=2$, it follows that $\left(x_{n}\right)_{n \in \mathbb{N}}$ converges to a point $\bar{x} \in$ Fix $T_{C_{1}, C_{2}}$. Using [20, Equation (22)] implies the existence of a point $c \in P_{C_{1}}(\bar{x})$ such that $c \in P_{C_{2}}(2 c-\bar{x})$. Consequently, $c \in C_{1} \cap C_{2}$ which completes the proof.

In the following corollary, we deduce the corresponding convergence result for the method of cyclic projections by observing that it can be cast as a special instance of the cyclically anchored DouglasRachford method.

Corollary 6.2 (Method of cyclic projections). Let $J:=\{1, \ldots, m\}$ and let $\left\{C_{j}\right\}_{j \in J}$ be a finite collection of union convex sets in $X$ with $x^{*} \in \cap_{j \in J} C_{j}$. Define a sequence $\left(x_{n}\right)_{n \in \mathbb{N}}$ with starting point $x_{0} \in X$ according to

$$
\forall n \in \mathbb{N}, \quad x_{n+1} \in P_{C_{i_{n}}}\left(x_{n}\right), \text { where } i_{n}=(n \bmod m)+1 .
$$

Then there exists an $r>0$ such that $\left(x_{n}\right)_{n \in \mathbb{N}}$ converges to a point $\bar{x} \in \cap_{j \in J} C_{j}$ whenever $x_{0} \in \operatorname{int} \mathbb{B}\left(x^{*} ; r\right)$.

Proof. We first note that, for a nonempty closed set $C, T_{X, C}=P_{C}$ and Fix $P_{C}=$ Fix $P_{C}=C$. The result now follows by applying Theorem 6.2 to $\left\{X, C_{1}, \ldots, C_{m}\right\}$; a collection of union convex sets with the first set, $X$, being convex.

Remark 6.2 (Sparse affine feasibility). Given a matrix $A$, point $b \in \operatorname{range}(A)$ and a sparsity bound $s$, the sparse affine feasibility problem asks for a point $x$ such that $A x=b$ and $\|x\|_{s} \leq s$. The method of alternating projections (i.e., Corollary 6.2 with $m=2$ ) applied to this problem has been studied by [21] who used regularity notions to show local linear convergence of the method of alternating projections. Whilst our Corollary 6.2 does applied to deduce local convergence for this problem, it does not say anything about the rate. 
Remark 6.3 (cyclic Douglas-Rachford method). It is not clear if the conclusions of Theorem 6.1 can be improved for the cyclic Douglas-Rachford method, specially, if it can be shown projectors of the limit $\bar{x}$ can be used to produce a point in the intersection $\cap_{j \in J} \cap C_{j}$, as in the case in the convex setting [17]. To illustrate the difficulty, consider the case when $J=\{1,2,3\}$. In this case, Theorem 6.1 gives that the limit $\bar{x}$ satisfies $\bar{x} \in \operatorname{Fix}\left(T_{C_{3}, C_{1}} \circ T_{C_{2}, C_{3}} \circ T_{C_{1}, C_{2}}\right)$. From this it is only possible to deduce that there exists convex subsets $C_{j}^{\prime}, C_{j}^{\prime \prime} \subseteq C_{j}$, for $j \in J$, with

$$
\bar{x} \in \operatorname{Fix}\left(T_{C_{3}^{\prime \prime}, C_{1}^{\prime \prime}} \circ T_{C_{2}^{\prime \prime}, C_{3}^{\prime}} \circ T_{C_{1}^{\prime}, C_{2}^{\prime}}\right),
$$

but where it is not necessarily the case that $C_{j}^{\prime}=C_{j}^{\prime \prime}$.

\subsection{The proximal point algorithm}

In this section, we consider the minimization problem

$$
\min _{x \in X} g(x)
$$

where $g: X \rightarrow(-\infty,+\infty]$ is min-convex.

Let $\gamma>0$. Given $x_{0} \in X$, the proximal point algorithm (with fixed stepsize) for (20) is given by

$$
\forall n \in \mathbb{N}, \quad x_{n+1} \in T_{\mathrm{PPA}}\left(x_{n}\right):=\operatorname{prox}_{\gamma g}\left(x_{n}\right) .
$$

By applying our main convergence result, we are able to deduce the following result regarding convergence of the proximal point algorithm the min-convex function $g$.

Theorem 6.3 (Proximal point algorithm). Let $g: X \rightarrow(-\infty,+\infty]$ be a min-convex function. Suppose that $x^{*} \in \mathbf{F i x} T_{\mathrm{PPA}}$. Denote $r:=r\left(x^{*} ; T_{\mathrm{PPA}}\right) \in(0,+\infty]$ and consider a sequence $\left(x_{n}\right)_{n \in \mathbb{N}}$ given by $(21)$ with $x_{0} \in \operatorname{int} \mathbb{B}\left(x^{*} ; r\right)$. Then $\left(x_{n}\right)_{n \in \mathbb{N}}$ converges to a local minimum of $g$.

Proof. By Proposition 5.2(f), the operator $T_{\mathrm{PPA}}$ is union 1/2-averaged nonexpansive. Consequently, convergence of the sequence $\left(x_{n}\right)_{n \in \mathbb{N}}$ to a point in Fix $T_{\mathrm{PPA}}$ then follows from Corollary 4.2 (with $\lambda_{n}=1$ ). The fact that every fixed point of $T_{\mathrm{PPA}}$ is a local minimum of $g$ follows from Proposition 5.2(e).

\subsection{Forward-backward splitting}

In this section, we consider the minimization problem

$$
\min _{x \in X}\{f(x)+g(x)\}
$$

where $f: X \rightarrow \mathbb{R}$ is a convex function with $L$-Lipschitz continuous gradient $\nabla f$, and $g:=\min _{i \in I} g_{i}: X \rightarrow$ $(-\infty,+\infty]$ is a min-convex function.

Given $x_{0} \in X$, the forward-backward algorithm for (22) is given by fixed point iteration

$$
\forall n \in \mathbb{N}, \quad x_{n+1} \in\left(1-\lambda_{n}\right) x_{n}+\lambda_{n} T_{\mathrm{FB}}\left(x_{n}\right) \text { with } T_{\mathrm{FB}}:=\operatorname{prox}_{\gamma g}(\operatorname{Id}-\gamma \nabla f),
$$

where $\gamma \in(0,2 / L)$ and $\left(\lambda_{n}\right)_{n \in \mathbb{N}} \subseteq(0,(4-\gamma L) / 2]$.

Remark 6.4. In the special case when $g_{i}$ are indicator functions to convex sets, the proximity operator $\operatorname{prox}_{\gamma g}$ reduces to a projection operators, and the corresponding algorithm is sometimes called the projected gradient algorithm. A specific example of which was studied in [5] arising is sparsity constrained minimization.

To begin, we study some properties of the forward-backward operator.

Proposition 6.2 (Properties of $T_{\mathrm{FB}}$ ). Let $f: X \rightarrow \mathbb{R}$ be a convex function with L-Lipscthiz continuous gradient, $g:=\min _{i \in I} g_{i}: X \rightarrow(-\infty,+\infty]$ be a min-convex function, and $\gamma \in(0,2 / L)$. Then the following assertions hold.

(a) The forward-backward splitting operator, $T_{\mathrm{FB}}$, is union $2 /(4-\gamma L)$-averaged nonexpansive.

(b) If $x \in \operatorname{Fix} T_{\mathrm{FB}}$, then there exists an $i \in I$ such that $0 \in\left(\nabla f(x)+\partial_{p} g(x)\right) \cap\left(\nabla f(x)+\partial g_{i}(x)\right)$.

(c) $x \in \operatorname{Fix} T_{\mathrm{FB}}$ if and only if there exists an $i \in I$ such that $x \in \arg \min \left\{f+g_{i}\right\}$ and ${ }^{\gamma} g(x-\gamma \nabla f(x))=$ ${ }^{\gamma} g_{i}(x-\gamma \nabla f(x))$, in which case $g(x)=g_{i}(x)$. 
(d) $x \in \mathbf{F i x} T_{\mathrm{FB}}$ if and only if $x \in \arg \min \left\{f+g_{i}\right\}$ for all $i \in I$ such that ${ }^{\gamma} g(x-\gamma \nabla f(x))={ }^{\gamma} g_{i}(x-$ $\gamma \nabla f(x))$, in which case $g(x)=g_{i}(x)$.

(e) $\operatorname{Fix} T_{\mathrm{FB}} \subseteq\{x \in X: x$ is a local minimum of $f+g\} \cap \operatorname{Fix} T_{\mathrm{FB}}$.

(f) If $x^{*} \in \mathbf{F i x} T_{\mathrm{FB}}$, then there exists a $\delta>0$ such that

$$
\operatorname{Fix} T_{\mathrm{FB}} \cap \mathbb{B}\left(x^{*} ; \delta\right) \subseteq\{x \in X: x \text { is a local minimum of } f+g\} .
$$

Proof. (a): First, by [3, Proposition 4.39 and Theorem 18.15(i)\&(v)], Id $-\gamma \nabla f$ is $(\gamma L) / 2$-averaged nonexpansive and, by Proposition 5.2(f), $\operatorname{prox}_{\gamma g}$ is union 1/2-averaged nonexpansive, in particular,

$$
\operatorname{prox}_{\gamma g}(x)=\left\{\operatorname{prox}_{\gamma g_{i}}(x): i \in \varphi(x)\right\},
$$

where $\varphi(x):=\left\{i \in I:{ }^{\gamma} g_{i}(x)={ }^{\gamma} g(x)\right\}$. Applying Proposition 3.2(c) it follows that $T_{\mathrm{FB}}$ is union $2 /(4-\gamma L)$-averaged nonexpansive with

$$
T_{\mathrm{FB}}(x)=\left\{\operatorname{prox}_{\gamma g_{i}}(x-\gamma \nabla f(x)): i \in \phi(x\},\right.
$$

where $\phi:=\varphi \circ(\mathrm{Id}-\gamma \nabla f)$ is osc.

(b): Let $x \in \operatorname{Fix} T_{\mathrm{FB}}$. Then $x \in \operatorname{prox}_{\gamma g}(x-\gamma \nabla f(x))$. Applying Proposition 5.1(c) to prox ${ }_{\gamma g}$ gives $\frac{1}{\gamma}(x-\gamma \nabla f(x)-x) \in \partial_{p} g(x)$ which implies that $0 \in \nabla f(x)+\partial_{p} g(x)$. Further, Proposition 5.2(f) implies that there exists an $i \in I$ such that $x=\operatorname{prox}_{\gamma_{g_{i}}}(x-\gamma \nabla f(x))$ and hence $0 \in \nabla f(x)+\partial g_{i}(x)$.

(c): Let $x \in X$ and set $y:=x-\gamma \nabla f(x)$. Then $x \in$ Fix $T_{\mathrm{FB}}$ if and only if there exists an $i \in I$ such that ${ }^{\gamma} g(y)={ }^{\gamma} g_{i}(y)$ and $x=\operatorname{prox}_{\gamma g_{i}}(y)$. Since $f, g_{i}$ are proper, lsc and convex and $f$ has full domain, the latter is equivalent to $x \in \arg \min \left\{f+g_{i}\right\}$ [3, Corollary 27.3(i)\&(viii)], thus establishing the claimed equivalence.

To prove the second claim, we note that $x=\operatorname{prox}_{\gamma g_{i}}(y) \in \operatorname{prox}_{\gamma g}(y)$ and ${ }^{\gamma} g(y)={ }^{\gamma} g_{i}(y)$ implies

$$
g(x)={ }^{\gamma} g(y)-\frac{1}{2 \gamma}\|y-x\|^{2}={ }^{\gamma} g_{i}(y)-\frac{1}{2 \gamma}\|y-x\|^{2}=g_{i}(x),
$$

as was claimed.

(d): Let $x \in X$ and set $y:=x-\gamma \nabla f(x)$. Then $x \in \mathbf{F i x} T_{\mathrm{FB}}$ if and only if $x=\operatorname{prox}_{\gamma g_{i}}(y)$ for all $i \in I$ such that ${ }^{\gamma} g(y)={ }^{\gamma} g_{i}(y)$. The rest is analogous to that of (c).

(e): We always have $\mathbf{F i x} T_{\mathrm{FB}} \subseteq \operatorname{Fix} T_{\mathrm{FB}}$. Let $x \in \mathbf{F i x} T_{\mathrm{FB}}$ and set $y:=x-\gamma \nabla f(x)$. It follows from (d) that

$$
\forall i \in I, \quad{ }^{\gamma} g(y)={ }^{\gamma} g_{i}(y) \Longrightarrow x \in \arg \min \left\{f+g_{i}\right\} .
$$

We shall prove that $x$ is a local minimum of $f+g$. Following Proposition 5.2(d) and (25), it suffices to show that

$$
\forall i \in I, \quad g(x)=g_{i}(x) \Longrightarrow{ }^{\gamma} g(y)={ }^{\gamma} g_{i}(y) .
$$

Indeed, let $i \in I$ be arbitrary such that $g(x)=g_{i}(x)$. Since $x \in \operatorname{prox}_{\gamma g}(y)$, using Proposition 5.2(a), we have

$$
{ }^{\gamma} g_{i}(y) \geq{ }^{\gamma} g(y)=g(x)+\frac{1}{2 \gamma}\|x-y\|^{2}=g_{i}(x)+\frac{1}{2 \gamma}\|x-y\|^{2} \geq{ }^{\gamma} g_{i}(y),
$$

which implies that ${ }^{\gamma} g(y)={ }^{\gamma} g_{i}(y)$.

(f): Let $x^{*} \in \mathbf{F i x} T_{\mathrm{FB}}$. Using (e), there exists $\delta_{1}>0$ such that $x^{*}$ is a minimum of $f+g$ on $\mathbb{B}\left(x^{*} ; \delta_{1}\right)$. By Proposition 4.1, there exists $\delta_{2}>0$ such that

$$
\forall x \in \mathbb{B}\left(x^{*} ; \delta_{2}\right), \quad \phi(x) \subseteq \phi\left(x^{*}\right) .
$$

Now take $\delta \in\left(0, \min \left\{\delta_{1}, \delta_{2}\right\}\right)$ and let $\bar{x} \in \operatorname{Fix} T_{\mathrm{FB}} \cap \mathbb{B}\left(x^{*} ; \delta\right)$. Then, by (c), there exists $i \in \phi(\bar{x})$ such that $\bar{x} \in \arg \min \left\{f+g_{i}\right\}$ and $g(\bar{x})=g_{i}(\bar{x})$. As $x^{*} \in \mathbf{F i x} T_{\mathrm{FB}}$ and $i \in \phi(\bar{x}) \subseteq \phi\left(x^{*}\right)$, we derive from (d) that $x^{*} \in \arg \min \left\{f+g_{i}\right\}$ and $g\left(x^{*}\right)=g_{i}\left(x^{*}\right)$. Therefore,

$$
f(\bar{x})+g(\bar{x})=f(\bar{x})+g_{i}(\bar{x})=f\left(x^{*}\right)+g_{i}\left(x^{*}\right)=f\left(x^{*}\right)+g\left(x^{*}\right) .
$$

Since $\bar{x} \in B\left(x^{*} ; \delta\right) \subset \operatorname{int} \mathbb{B}\left(x^{*} ; \delta_{1}\right)$ with the same value as $f+g$ at $x^{*}, \bar{x}$ is also a local minimum of $f+g$. 
Our main results regarding convergence of the forward-backward method can now be stated as follows.

Theorem 6.4 (Forward-backward splitting). Let $f: X \rightarrow \mathbb{R}$ be a convex function with L-Lipscthiz continuous gradient, $g: X \rightarrow(-\infty,+\infty]$ be a min-convex function, and $\gamma \in(0,2 / L)$. Suppose that $x^{*} \in \mathbf{F i x} T_{\mathrm{FB}}$ and let $\left(\lambda_{n}\right)_{n \in \mathbb{N}}$ be a sequence in $\left(0, \frac{4-\gamma L}{2}\right]$ with $\liminf _{n \rightarrow \infty} \lambda_{n}\left(\frac{4-\gamma L}{2}-\lambda_{n}\right)>0$. Denote $r:=r\left(x^{*} ; T_{\mathrm{FB}}\right) \in(0,+\infty]$ and consider a sequence $\left(x_{n}\right)_{n \in \mathbb{N}}$ given by $(23)$ with $x_{0} \in \operatorname{int} \mathbb{B}\left(x^{*} ; r\right)$. Then $\left(x_{n}\right)_{n \in \mathbb{R}}$ converges to a point in $\bar{x} \in \mathrm{Fix} T_{\mathrm{FB}} \cap \mathbb{B}\left(x^{*} ; r\right)$. Furthermore, for sufficiently small $r>0$, the limit point $\bar{x}$ is a local minimum of $f+g$.

Proof. Convergence of the sequence $\left(x_{n}\right)_{n \in \mathbb{N}}$ to a point $\bar{x} \in \mathrm{Fix} T_{\mathrm{FB}} \cap \mathbb{B}\left(x^{*} ; r\right)$ follows by combining Proposition 6.2(a) and Corollary 4.2. For sufficiently small $r>0$, the fact that $\bar{x}$ is a local minimum of $f+g$ follows from Proposition 6.2(f).

In the proof of the previous theorem, we used an inclusion relating fixed points and local minima in Proposition 6.2(f). To conclude our study of the forward-backward method, we show that osc representability gives the reverse inclusion.

Proposition 6.3 (Local minima are fixed points). Let $f: X \rightarrow \mathbb{R}$ be convex with L-Lipscthiz continuous gradient, let $g:=\min _{i \in I} g_{i}: X \rightarrow(-\infty,+\infty]$ be min-convex and osc representable at $\bar{x} \in X$. Suppose that $\inf (f+g)(X)>-\infty$ and that $\bar{x}$ is a local minimum of $f+g$. There exist constants $\bar{\gamma}, \delta>0$ such that, for each $x \in \mathbb{B}(\bar{x} ; \delta)$ and $\gamma \in(0, \bar{\gamma}]$, there exists an index $i \in I$ satisfying:

(a) $\bar{x} \in \arg \min \left\{f+g_{i}\right\}$,

(b) ${ }^{\gamma} g(x-\gamma \nabla f(x))={ }^{\gamma} g_{i}(x-\gamma \nabla f(x))$, and

(c) $\operatorname{prox}_{\gamma g_{i}}(x-\gamma \nabla f(x)) \in \operatorname{prox}_{\gamma g}(x-\gamma \nabla f(x))$.

Consequently, $\bar{x} \in \operatorname{Fix} T_{\mathrm{FB}}$ for all $\gamma \in(0, \bar{\gamma}]$.

Proof. Assume that $\bar{x}$ is a local minimum of $f+g$ and consider the selector $\phi: X \rightrightarrows I$ defined by

$$
\phi(x):=\left\{i \in I: g(x)=g_{i}(x)\right\} .
$$

Then, by Proposition 5.2(d), $\bar{x} \in \arg \min \left\{f+g_{i}\right\}$ for all $i \in \phi(\bar{x})$. Since $\phi$ is osc at $\bar{x}$ by assumption, Proposition 4.1 implies that there exists $\delta>0$ such that

$$
\forall x \in \mathbb{B}(\bar{x} ; 3 \delta), \quad \phi(x) \subseteq \phi(\bar{x})
$$

Fix a constant $\bar{\gamma} \in\left(0, \frac{1}{2 L}\right)$ satisfying

$$
2 \bar{\gamma}((f+g)(\bar{x})-\inf (f+g)(X))<\delta^{2} .
$$

Let $\gamma \in(0, \bar{\gamma}], x \in \mathbb{B}(\bar{x} ; \delta)$ and $z \in \operatorname{prox}_{\gamma g}(x-\gamma \nabla f(x))$. Then

$$
g(\bar{x})+\frac{1}{2 \gamma}\|x-\gamma \nabla f(x)-\bar{x}\|^{2} \geq \gamma g(x-\gamma \nabla f(x))=g(z)+\frac{1}{2 \gamma}\|x-\gamma \nabla f(x)-z\|^{2} .
$$

Since $f$ is convex with $L$-Lipschitz continuous gradient, [3, Proposition 17.7(ii) and Theorem 18.15(iii)] implies that

$$
f(z) \leq f(\bar{x})+\langle\nabla f(x), z-\bar{x}\rangle+\frac{L}{2}\|z-x\|^{2} .
$$

Combining the previous two equations gives

$$
\begin{aligned}
2 \gamma(g(\bar{x})-g(z)) & \geq\|x-\gamma \nabla f(x)-z\|^{2}-\|x-\gamma \nabla f(x)-\bar{x}\|^{2} \\
& =\|x-z\|^{2}-\|x-\bar{x}\|^{2}+2 \gamma\langle\nabla f(x), z-\bar{x}\rangle \\
& \geq\|x-z\|^{2}-\|x-\bar{x}\|^{2}+2 \gamma\left(f(z)-f(\bar{x})-\frac{L}{2}\|z-x\|^{2}\right)
\end{aligned}
$$

which together with (27) yields

$$
\delta^{2}>2 \gamma((f+g)(\bar{x})-\inf (f+g)(X)) \geq(1-\gamma L)\|x-z\|^{2}-\|x-\bar{x}\|^{2} \geq \frac{1}{2}\|x-z\|^{2}-\delta^{2} .
$$


We obtain that $\|x-z\| \leq 2 \delta$ and so $\|z-\bar{x}\| \leq\|z-x\|+\|x-\bar{x}\| \leq 3 \delta$. Therefore, $z \in \mathbb{B}(\bar{x} ; 3 \delta)$ and, by (26), $\phi(z) \subseteq \phi(\bar{x})$. Take any $i \in \phi(z)$. On the one hand, $i \in \phi(\bar{x})$, and so $\bar{x} \in \arg \min \left\{f+g_{i}\right\}$. On the other hand, $g(z)=g_{i}(z)$, which implies that

$$
\begin{aligned}
\gamma g(x-\gamma \nabla f(x)) & =g(z)+\frac{1}{2 \gamma}\|x-\gamma \nabla f(x)-z\|^{2} \\
& =g_{i}(z)+\frac{1}{2 \gamma}\|x-\gamma \nabla f(x)-z\|^{2} \\
& \geq{ }^{\gamma} g_{i}(x-\gamma \nabla f(x)) \geq{ }^{\gamma} g(x-\gamma \nabla f(x)) .
\end{aligned}
$$

It follows that ${ }^{\gamma} g(x-\gamma \nabla f(x))={ }^{\gamma} g_{i}(x-\gamma \nabla f(x))$ and also $\operatorname{prox}_{\gamma g_{i}}(x-\gamma \nabla f(x)) \in \operatorname{prox}_{\gamma g}(x-\gamma \nabla f(x))$.In particular, ${ }^{\gamma} g(\bar{x}-\gamma \nabla f(\bar{x}))={ }^{\gamma} g_{i}(\bar{x}-\gamma \nabla f(\bar{x}))$ and hence, by appealing to Proposition $6.2(\mathrm{c})$, we deduce that $\bar{x} \in \operatorname{Fix} T_{\mathrm{FB}}$.

\subsection{Douglas-Rachford splitting}

In this section, we consider the minimization problem

$$
\min _{x \in X}\{f(x)+g(x)\}
$$

where $f: X \rightarrow(-\infty,+\infty]$ and $g: X \rightarrow(-\infty,+\infty]$ are min-convex functions.

Given $x_{0} \in X$ and sequence $\left(\lambda_{n}\right)_{n \in \mathbb{N}} \subseteq(0,2]$, the Douglas-Rachford splitting algorithm for (28) can be described as iteration

$$
\forall n \in \mathbb{N}, \quad\left\{\begin{aligned}
y_{n} & \in \operatorname{prox}_{\gamma f}\left(x_{n}\right) \\
z_{n} & \in \operatorname{prox}_{\gamma g}\left(2 y_{n}-x_{n}\right), \\
x_{n+1} & =x_{n}+\lambda_{n}\left(z_{n}-y_{n}\right) .
\end{aligned}\right.
$$

In the case when $f$ and $g$ are convex, this is just the the usual Douglas-Rachford splitting algorithm for the sum convex functions. The iteration (29) can be cast as a fixed point iteration in the sequence $\left(x_{n}\right)_{n \in \mathbb{N}}$. Precisely, it may be expressed as

$$
\forall n \in \mathbb{N}, \quad x_{n+1} \in\left(1-\lambda_{n}\right) x_{n}+\lambda_{n} T_{\mathrm{DR}}\left(x_{n}\right),
$$

where $T_{\mathrm{DR}}$ is the Douglas-Rachford splitting operator defined by

$$
\begin{aligned}
T_{\mathrm{DR}}(x): & =\frac{1}{2}\left(\mathrm{Id}+\left(2 \operatorname{prox}_{\gamma g}-\mathrm{Id}\right) \circ\left(2 \operatorname{prox}_{\gamma f}-\mathrm{Id}\right)\right)(x) \\
& =\left\{x+z-y: y \in \operatorname{prox}_{\gamma f}(x), z \in \operatorname{prox}_{\gamma g}(2 y-x)\right\}
\end{aligned}
$$

In the special case when the functions $f_{j}$ and $g_{i}$ are the indicator functions to convex sets, this reduces to the Douglas-Rachford projection algorithms considered in Section 6.1.

We begin by examining properties of the underlying operator $T_{\mathrm{DR}}$.

Proposition 6.4 (Properties of $\left.T_{\mathrm{DR}}\right)$. Let $f: X \rightarrow(-\infty,+\infty]$ and $g: X \rightarrow(-\infty,+\infty]$ be proper functions and let $\gamma>0$. Then the following assertions hold.

(a) $x \in \operatorname{Fix} T_{\mathrm{DR}}$ if and only if there exists $y \in \operatorname{prox}_{\gamma f}(x)$ such that $y \in \operatorname{prox}_{\gamma g}(2 y-x)$.

(b) $x \in \mathbf{F i x} T_{\mathrm{DR}}$ if and only if $\{y\}=\operatorname{prox}_{\gamma g}(2 y-x)$ for all $y \in \operatorname{prox}_{\gamma f}(x)$.

(c) If $x \in \operatorname{Fix} T_{\mathrm{DR}}$, then there exists $y \in \operatorname{prox}_{\gamma f}(x)$ such that $0 \in \partial_{p} f(y)+\partial_{p} g(y) \subseteq \partial_{p}(f+g)(y)$.

(d) If $f$ and $g$ are min-convex, then $T_{\mathrm{DR}}$ is union $1 / 2$-averaged nonexpansive.

Proof. (a) \& (b): This follows from (30).

(c): Let $x \in \operatorname{Fix} T_{\mathrm{DR}}$. Using (a), there exists $y \in \operatorname{prox}_{\gamma f}(x)$ such that $y \in \operatorname{prox}_{\gamma g}(2 y-x)$. From Proposition 5.1(c), we deduce that

$$
\frac{1}{\gamma}(x-y) \in \partial_{p} f(y) \text { and } \frac{1}{\gamma}((2 y-x)-y)=\frac{1}{\gamma}(y-x) \in \partial_{p} g(y) .
$$

Summing these expressions gives $0 \in \partial_{p} f(y)+\partial_{p} g(y) \subseteq \partial_{p}(f+g)(y)$, which proves the claim.

(d): By Proposition 5.2(f), both $\operatorname{prox}_{\gamma f}$ and $\operatorname{prox}_{\gamma g}$ are union 1/2-averaged nonexpansive. Applying Proposition 3.1 with $\alpha=1 / 2$ implies that both $2 \operatorname{prox}_{\gamma f}-\mathrm{Id}$ and $2 \operatorname{prox}_{\gamma g}-$ Id are union nonexpansive, and hence so is $\left(2 \operatorname{prox}_{\gamma g}-\mathrm{Id}\right) \circ\left(2 \operatorname{prox}_{\gamma f}-\mathrm{Id}\right)$ due to Proposition 3.2(c). Again applying Proposition 3.1 with $\alpha=1 / 2$ completes the proof. 
Proposition 6.5 (Finer properties of $\left.T_{\mathrm{DR}}\right)$. Let $f: X \rightarrow \mathbb{R}$ be convex, $g:=\min _{i \in I} g_{i}: X \rightarrow(-\infty,+\infty]$ be min-convex function, and $\gamma>0$. Then the following assertions hold.

(a) $x \in \operatorname{Fix} T_{\mathrm{DR}}$ if and only if there exists an $i \in I$ such that $y:=\operatorname{prox}_{\gamma f}(x) \in \arg \min \left\{f+g_{i}\right\}$ and ${ }^{\gamma} g(2 y-x)={ }^{\gamma} g_{i}(2 y-x)$. Moreover any such point $y$ satisfies $g(y)=g_{i}(y)$.

(b) $x \in \mathbf{F i x} T_{\mathrm{DR}}$ if and only if $y:=\operatorname{prox}_{\gamma f}(x) \in \arg \min \left\{f+g_{i}\right\}$ for all $i \in I$ such that ${ }^{\gamma} g(2 y-x)=$ ${ }^{\gamma} g_{i}(2 y-x)$. Moreover, the point $y$ satisfies $g(y)=g_{i}(y)$.

(c) If $x \in \mathbf{F i x} T_{\mathrm{DR}}$, then $\operatorname{prox}_{\gamma f}(x)$ is a local minimum of $f+g$.

(d) If $x^{*} \in \mathbf{F i x} T_{\mathrm{DR}}$, then there exists a $\delta>0$ such that

$$
\operatorname{prox}_{\gamma f}\left(\operatorname{Fix} T_{\mathrm{DR}} \cap \mathbb{B}\left(x^{*} ; \delta\right)\right) \subseteq\{x \in X: x \text { is a local minimum of } f+g\} .
$$

Proof. We first note that $\operatorname{prox}_{\gamma f}$ is single-valued by [3, Proposition 12.15] and, by Proposition 5.2, that

$$
\operatorname{prox}_{\gamma g}(x)=\left\{\operatorname{prox}_{\gamma g_{i}}(x): i \in I,{ }^{\gamma} g_{i}(x)={ }^{\gamma} g(x)\right\} .
$$

(a): Let $x \in X$ and denote $y:=\operatorname{prox}_{\gamma f}(x)$. By Proposition 6.4(a), $x \in$ Fix $T_{\mathrm{DR}}$ if and only if

$$
y \in \operatorname{prox}_{\gamma g}(2 y-x)=\left\{\operatorname{prox}_{\gamma g_{i}}(2 y-x): i \in I,{ }^{\gamma} g(2 y-x)={ }^{\gamma} g_{i}(2 y-x)\right\} .
$$

This is equivalent to the existence of an index $i \in I$ such that $y=\operatorname{prox}_{\gamma g_{i}}(2 y-x)$ and ${ }^{\gamma} g(2 y-x)=$ ${ }^{\gamma} g_{i}(2 y-x)$. Since $f$ has full domain, by applying [3, Corollary 27.3(i)\&(iii)] to $f$ and $g_{i}$, we deduce that

$$
y=\operatorname{prox}_{\gamma f}(x)=\operatorname{prox}_{\gamma g_{i}}(2 y-x) \Longleftrightarrow y \in \arg \min \left\{f+g_{i}\right\} .
$$

Moreover, it follows from $y=\operatorname{prox}_{\gamma g_{i}}(2 y-x) \in \operatorname{prox}_{\gamma g}(2 y-x)$ and ${ }^{\gamma} g(2 y-x)={ }^{\gamma} g_{i}(2 y-x)$ that

$$
g(y)={ }^{\gamma} g(2 y-x)-\frac{1}{2 \gamma}\|y-x\|^{2}={ }^{\gamma} g_{i}(2 y-x)-\frac{1}{2 \gamma}\|y-x\|^{2}=g_{i}(y),
$$

which completes the claim.

(b): Let $x \in X$ and denote $y:=\operatorname{prox}_{\gamma f}(x)$. By Proposition 6.4(b), $x \in \mathbf{F i x} T_{\mathrm{DR}}$ if and only if

$$
\{y\}=\operatorname{prox}_{\gamma g}(2 y-x)=\left\{\operatorname{prox}_{\gamma g_{i}}(2 y-x): i \in I,{ }^{\gamma} g(2 y-x)={ }^{\gamma} g_{i}(2 y-x)\right\},
$$

which is equivalent to $y=\operatorname{prox}_{\gamma g_{i}}(2 y-x)$ for all $i \in I$ such that ${ }^{\gamma} g(2 y-x)={ }^{\gamma} g_{i}(2 y-x)$. The remainder of the proof is similar to (a).

(c): Let $x \in \mathbf{F i x} T_{\mathrm{DR}}$ and denote $y:=\operatorname{prox}_{\gamma f}(x)$. Then, by (b),

$$
i \in I \text { and }{ }^{\gamma} g(2 y-x)={ }^{\gamma} g_{i}(2 y-x) \Longrightarrow y \in \arg \min \left\{f+g_{i}\right\} .
$$

Now, take any $i \in I$ satisfying $g(y)=g_{i}(y)$. Noting that $y \in \operatorname{prox}_{\gamma g}(2 y-x)$ and using Proposition 5.2(a), it follows that

$$
{ }^{\gamma} g(2 y-x)=g(y)+\frac{1}{2 \gamma}\|(2 y-x)-y\|^{2}=g_{i}(y)+\frac{1}{2 \gamma}\|(2 y-x)-y\|^{2} \geq{ }^{\gamma} g_{i}(2 y-x) \geq{ }^{\gamma} g(2 y-x),
$$

which yields ${ }^{\gamma} g(2 y-x)={ }^{\gamma} g_{i}(2 y-x)$. By (31), $y \in \arg \min \left\{f+g_{i}\right\}$. Since this holds for any $i \in I$ satisfying $g(y)=g_{i}(y)$, Proposition 5.2(d) implies that $y$ is a local minimum of $f+g$.

(d): Let $x^{*} \in \mathbf{F i x} T_{\mathrm{DR}}$ and set $y^{*}:=\operatorname{prox}_{\gamma f}\left(x^{*}\right)$. By (c), there exists $\delta_{1}>0$ such that $y^{*}$ is a minimum of $f+g$ on $\mathbb{B}\left(y^{*} ; \delta_{1}\right)$. Consider $\phi: X \rightrightarrows I$ given by

$$
\phi(x):=\left\{i \in I:{ }^{\gamma} g(2 y-x)={ }^{\gamma} g_{i}(2 y-x), y:=\operatorname{prox}_{\gamma f}(x)\right\} .
$$

Noting that $x \mapsto 2 \operatorname{prox}_{\gamma f}(x)-x$ is continuous, combining Propositions 2.4 and 5.3 shows that $\phi$ is osc. Consequently, Proposition 4.1 yields the existence of $\delta_{2}>0$ such that

$$
\forall x \in \mathbb{B}\left(x^{*} ; \delta_{2}\right), \quad \phi(x) \subseteq \phi\left(x^{*}\right) .
$$

Now take $\delta \in\left(0, \min \left\{\delta_{1}, \delta_{2}\right\}\right)$, let $\bar{x} \in \operatorname{Fix} T_{\mathrm{DR}} \cap \mathbb{B}\left(x^{*} ; \delta\right)$, and set $\bar{y}:=\operatorname{prox}_{\gamma f}(\bar{x})$. Using (a), there exists $i \in \phi(\bar{x})$ such that $\bar{y} \in \arg \min \left\{f+g_{i}\right\}$ and $g(\bar{y})=g_{i}(\bar{y})$. Since $x^{*} \in \mathbf{F i x} T_{\mathrm{DR}}$ and $i \in \phi(\bar{x}) \subseteq \phi\left(x^{*}\right)$, it also holds that $y^{*} \in \arg \min \left\{f+g_{i}\right\}$ and $g\left(y^{*}\right)=g_{i}\left(y^{*}\right)$ due to (b). We deduce that

$$
f(\bar{y})+g(\bar{y})=f(\bar{y})+g_{i}(\bar{y})=f\left(y^{*}\right)+g_{i}\left(y^{*}\right)=f\left(y^{*}\right)+g\left(y^{*}\right) .
$$

Moreover, $\bar{y} \in \mathbb{B}\left(y^{*} ; \delta\right) \subset \operatorname{int} \mathbb{B}\left(y^{*} ; \delta_{1}\right)$ since $\bar{x} \in B\left(x^{*} ; \delta\right)$ and $\operatorname{prox}_{\gamma f}$ is nonexpansive. Therefore, $\bar{y}$ is also a local minimum of $f+g$. 
Theorem 6.5 (Douglas-Rachford splitting). Let $f: X \rightarrow \mathbb{R}$ be convex, $g: X \rightarrow(-\infty,+\infty]$ be minconvex, and $\gamma>0$. Suppose that $x^{*} \in \mathbf{F i x} T_{\mathrm{DR}}$ and let $\left(\lambda_{n}\right)_{n \in \mathbb{N}}$ be a sequence in $(0,2]$ with $\liminf _{n \rightarrow \infty} \lambda_{n}(2-$ $\left.\lambda_{n}\right)>0$. Denote $r:=r\left(x^{*} ; T_{\mathrm{DR}}\right) \in(0,+\infty]$ and consider a sequence $\left(x_{n}\right)_{n \in \mathbb{N}}$ given by (29) with $x_{0} \in \operatorname{int} \mathbb{B}\left(x^{*} ; r\right)$. Then $\left(x_{n}\right)_{n \in \mathbb{R}}$ converges to a point $\bar{x} \in \operatorname{Fix} T_{\mathrm{DR}} \cap \mathbb{B}\left(x^{*} ; r\right)$. Furthermore, for sufficiently small $r>0, \operatorname{prox}_{\gamma f}(\bar{x})$ is a local minimum of $f+g$.

Proof. Convergence of the sequence $\left(x_{n}\right)_{n \in \mathbb{N}}$ to a fixed point, $\bar{x}$, follows by combining Proposition 6.4 (d) and Corollary 4.2. The remaining conclusion follows from Proposition 6.5(d).

\section{Conclusions}

In this work, we have introduced and studied the classes of union nonexpansive and union averaged nonexpansive operators. Fixed point iterations based on these operators are locally convergent to fixed points when initialized near strong fixed points. The convergence behavior of proximal algorithms applied to minimization problems involving min-convex functions can be systematically studied using the notion.

\section{Acknowledgments}

MND was partially supported by the Australian Research Council (ARC) Discovery Project DP160101537 and by the Priority Research Centre for Computer-Assisted Research Mathematics and its Applications (CARMA) at the University of Newcastle. He wishes to acknowledge the hospitality and the support of D. Russell Luke during his visit to the Universität Göttingen. MKT was partially supported by a Postdoctoral Fellowship from the Alexander von Humboldt Foundation.

\section{References}

[1] Cegielski, A.: Iterative Methods for Fixed Point Problems in Hilbert Spaces. Springer, Heidelberg (2012)

[2] Opial, Z.: Weak convergence of the sequence of successive approximation for nonexpansive mappings. Bull. Amer. Math. Soc. 73(4), 591-597 (1967)

[3] Bauschke, H.H., Combettes, P.L.: Convex Analysis and Monotone Operator Theory in Hilbert Spaces, 2nd edn. Springer, Cham (2017)

[4] Baillon, J.B., Bruck, R.E., Reich, S.: On the asymptotic behavior of nonexpansive mappings and semigroups in Banach spaces. Houston J. Math. 4(1), 1-9 (1978)

[5] Tam, M.K.: Algorithms based on unions of nonexpansive maps. Optim. Lett. 12(5), 1019-1027 (2018)

[6] Bauschke, H.H., Noll, D.: On the local convergence of the Douglas-Rachford algorithm. Arch. Math. 102(6), 589-600 (2014)

[7] Elsner, L., Koltracht, I., Neumann, M.: Convergence of sequential and asynchronous nonlinear paracontractions. Numer. Math. 62(3), 305-319 (1992)

[8] Combettes, P.L., Yamada, I.: Compositions and convex combinations of averaged nonexpansive operators. J. Math. Anal. Appl. 425(1), 55-70 (2015)

[9] Dontchev, A.L., Rockafellar, R.T.: Implicit Functions and Solution Mappings, 2nd edn. Springer, New York (2014)

[10] Bauschke, H.H., Lucet, Y., Phan, H.: On the convexity of piecewise-defined functions. ESAIM Control Optim. Calc. Var. 22(3), 728-742 (2016)

[11] Eberhard, A., Roshchina, V., Sang, T.: Outer limits of subdifferentials for min-max type functions. Optimization (2017). DOI 10.1080/02331934.2017.1398750

[12] Mordukhovich, B.S., Nam, N.M.: Exact calculus for proximal subgradients with applications to optimization. ESAIM Proc. 17, 80-95 (2007) 
[13] Clarke, F.: Functional Analysis, Calculus of Variations and Optimal Control. Springer, London (2013)

[14] Dao, M.N., Phan, H.M.: Linear convergence of projection algorithms. Math. Oper. Res. (2018). DOI 10.1287/moor.2018.0942

[15] Dao, M.N., Phan, H.M.: Linear convergence of the generalized Douglas-Rachford algorithm for feasibility problems. J. Global Optim. (2018). DOI 10.1007/s10898-018-0654-x

[16] Luke, D.R.: Finding best approximation pairs relative to a convex and prox-regular set in a Hilbert space. SIAM J. Optim. 19(2), 714-739 (2008)

[17] Borwein, J.M., Tam, M.K.: A cyclic Douglas-Rachford iteration scheme. J. Optim. Theory 160(1), $1-29(2014)$

[18] Borwein, J.M., Tam, M.K.: The cyclic Douglas-Rachford method for inconsistent feasibility problems. J. Nonlinear Convex Anal. 16(4), 537-587 (2015)

[19] Bauschke, H.H., Noll, D., Phan, H.: Linear and strong convergence of algorithms involving averaged nonexpansive operators. J. Math. Anal. and Appl. 421(1), 1-20 (2015)

[20] Bauschke, H.H., Dao, M.N.: On the finite convergence of the Douglas-Rachford algorithm for solving (not necessarily convex) feasibility problems in Euclidean spaces. SIAM J. Optim. 27(1), 507-537 (2017)

[21] Hesse, R., Luke, D.R., Neumann, P.: Alternating projections and Douglas-Rachford for sparse affine feasibility. IEEE Trans. Signal Process 62(18), 4868-4881 (2014) 